\title{
A Novel Coexistence Scheme for IEEE 802.11 for User Fairness and Efficient Spectrum Utilization in the Presence of LTE-U
}

\author{
Anand M. Baswade, Touheed Anwar Atif, Bheemarjuna Reddy Tamma, and Antony Franklin A \\ Department of Computer Science and Engineering, Indian Institute of Technology Hyderabad, India
}

\begin{abstract}
A promising solution satisfying the industry's demand to have minimum modification in LTE for its operation in unlicensed spectrum is duty cycled LTE-U scheme, which adopts discontinuous transmission to ensure fair coexistence with 802.11 (Wi-Fi) WLANs. Even though the scheme guarantees to maintain Wi-Fi network performance, the fairness among Wi-Fi users still remains arcane. In this work, we present a practical scenario where LTE-U, despite being discontinuous (by following an ON/OFF cycle), results in not only unfair throughput distribution among Wi-Fi users but also causes degradation in Wi-Fi APs downlink performance. This is due to the domination of few Wi-Fi users who harness channel in both ON and OFF durations of LTE-U, namely non-victim users over those who get access only in OFF duration, called victim users. In this paper, we studied the performance of victim and non-victim Wi-Fi users, and Wi-Fi AP while varying LTE-U ON fraction (i.e., duty cycle). A propitious scheme is proposed for WLANs, with regard to ease of implementation, employing Point/Hybrid Coordination Function (PCF/HCF) mode of 802.11, promising fairness among Wi-Fi users with improvement in the channel utilization of Wi-Fi network. The key idea is that the victim users, who can only be served during the LTE-U OFF period should be served in Contention Free Period (CFP) - so as to improve their throughputs and make them equally competitive with non-victim users. Also, we present an analytical model to demonstrate guaranteed improvement and to validate our simulation results.
\end{abstract}

Keywords: IEEE 802.11 (Wi-Fi), LTE in Unlicensed (LTE-U), Distributed Coordination Function (DCF), Point Coordination Function (PCF) and Coexistence Coordination Function (CCF)

\section{Introduction}

An incessant increase in mobile data traffic [1] demand with limited licensed spectrum has called upon the traditional telecom operators, to focus on the unlicensed spectrum as a promising solution. Fair coexistence with other technologies operating in unlicensed spectrum - mainly 802.11 (Wi-Fi) - needs to be ensured for successful deployment of LTE-U in different regulatory domains. Though the two schemes, Licensed Assisted Access (LAA) 2 i.e., LTE in Unlicensed with Listen Before Talk (LBT) 3 and duty cycled LTE-U 4 are shown to be equally fair with Wi-Fi 5, the need for quick deployment has forced LTE-U forum to narrow down its focus on markets promoting duty cycled LTE-U [6]. Duty cycled LTE-U is a simple scheme where LTE-U base station (eNB) follows duty cycle based discontinuous transmission approach to fairly share an unlicensed channel with Wi-Fi networks operating on the same channel. One such example is Carrier Sense Adaptive Transmission (CSAT) [7] where LTE-U eNB follows ON and OFF cycle pattern in a given duty

\footnotetext{
${ }^{1}$ Email addresses: cs14resch11002@iith.ac.in (Anand M. Baswade), ee13b1036@iith.ac.in (Touheed Anwar Atif), tbr@iith.ac.in (Bheemarjuna Reddy Tamma), antony.franklin@iith.ac.in (Anotny Franklin A)
} 


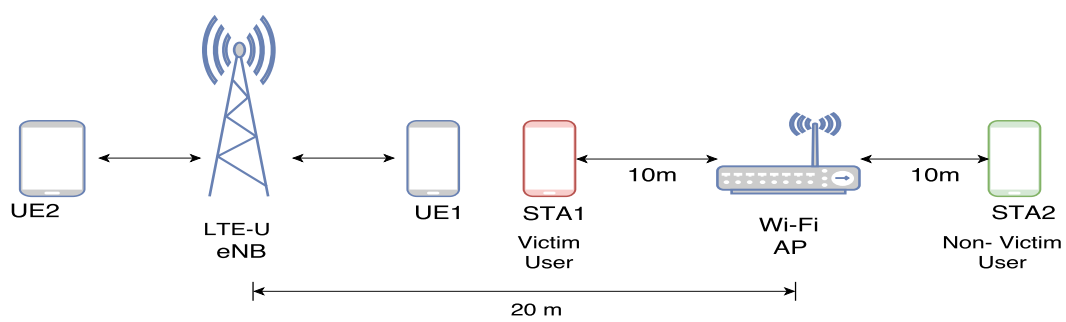

Figure 1: Motivational example scenario with STA1 and STA2 as Wi-Fi users. STA1 is a victim user because the interference from LTE-U causes its $S I N R$ to decrease below a minimum required $S I N R$ in LTE-U ON period.

cycle period, with ON duration corresponding to LTE-U operation meant to serve LTE-U users in downlink (LTE-U uses unlicensed spectrum only in downlink [6]) and OFF duration corresponding to LTE-U notransmission, to allow Wi-Fi operation. This works well when Wi-Fi AP, the center of communication in an infrastructure Basic Service Set (BSS), is also affected by LTE-U transmissions and hence can serve its users only in LTE-U OFF duration. But, in a scenario as shown in Fig. 1, where Wi-Fi AP and some Wi-Fi users can transmit or receive packets in LTE-U duty cycle (i.e., LTE-U ON period), while remaining users cannot transmit or receive, the channel access and consequently the throughput among Wi-Fi users become unfair. A similar scenario is considered in [8] where the authors presented an intercell interference coordination technique when two LTE-U eNBs interfere, but managing in a scenario when LTE-U eNB interferes with some users of a Wi-Fi network is unprecedented and is considered as part of our study in this paper.

Fig. 1 shows the scenario where LTE-U eNB is hidden from Wi-Fi AP hence, the effect of LTE-U on Wi-Fi AP is not to an extent that Wi-Fi AP has to defer from channel access during the LTE-U ON period, implying that it can operate in both the LTE-U ON and OFF periods. A Wi-Fi network following Distributed Coordination Function (DCF) mode of operation (referred as DCF mechanism for rest of the paper) ensures that every Wi-Fi user has equal chance to access the channel, including the Wi-Fi AP — which is what happens during LTE-U OFF period. But during LTE-U ON period, Wi-Fi users affected by LTE-U, fail to successfully transmit or receive any data due to one of the following two reasons: i) because they sense channel is busy due to LTE-U transmissions and ii) the interference from LTE-U decreases their Signal to Interference plus Noise Ratio $(S I N R)$, below a minimum $S I N R$ required for successful reception. Note that the successful transmissions in a Wi-Fi network are only the ones which are successfully received and acknowledged, failure to receive a packet or even acknowledge a successfully received packet would eventually be considered as an unsuccessful transmission. We call these users as victim users as they can not transmit and receive packets in LTE-U ON period. On the other hand, remaining users, called as non-victim users, continue to transmit and receive, even during the LTE-U ON period and thus dominate the victim users. In Fig. 1. STA1 is a victim user while STA2 is a non-victim user. This domination of non-victim users during the LTE-U ON period causes severe unfairness among the victim and non-victim users in Wi-Fi network.

Also, every transmission attempt of Wi-Fi AP to the victim users, during the LTE-U ON period fails and thus elicits multiple retransmissions of the same packet leading to wastage of the channel resources. Hence, our focus in this work, mainly lies in improving the fairness and channel utilization in the above scenario by enhancing the throughput of victim users and Wi-Fi AP irrespective of LTE-U duty cycle.

The main contributions of the paper can be summarized as follows:

- We study the considered hidden terminal scenario in detail and analyze the performance of Wi-Fi network, and its users in the presence of duty cycled LTE-U. We observe the unfairness caused to the victim users in terms of throughput and also study the effect of presence of these victim users on a Wi-Fi network.

- We propose a solution which is easy to adopt; the proposed solution can be adopted to achieve Wi-Fi user fairness and to improve the performance of Wi-Fi network. 
- We propose an analytical model that shows the guaranteed improvement of the proposed scheme.

- Finally, to demonstrate the efficacy of the proposed scheme, we evaluate its performance through analysis and validate it through simulation results.

The rest of the paper is organized as follows. Section II gives a motivational example for this work. Related work is provided in Section III, and Section IV describes the system model. Proposed work is discussed in Section V. Section VI gives performance analysis of proposed work, Section VII presents the experimental setup and results showing guaranteed improvement of the proposed scheme. Finally, Section VIII summarizes and concludes the work.

\section{Motivational Example}
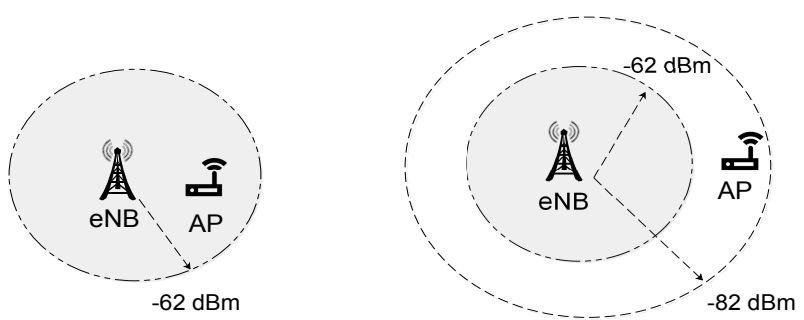

Figure 2: Two cases when Wi-Fi AP receives the signal with interference power more than $-62 \mathrm{dBm}$ and less than $-62 \mathrm{dBm}$ from LTE-U eNB.

A received interference power from LTE-U eNB at Wi-Fi AP can be more than $-62 \mathrm{dBm}$ (i.e., Energy Detection Threshold (EDT)) or less than $-62 \mathrm{dBm}$ as shown in Fig. 2. If received interference power at Wi-Fi AP from LTE-U eNB is more than $-62 \mathrm{dBm}$, then AP can defer access to the medium during LTE-U ON period. In such scenarios, AP gets access to the medium only in LTE-U OFF period, and thus it serves all users in LTE-U OFF period only. Hence, all users perform equally which results in the user fairness. But if received interference power at Wi-Fi AP from LTE-U eNB is less than -62 dBm then AP can transmit in LTE-U ON as well as LTE-U OFF periods where it can serve only a few users in ON period but can serve all users in LTE-U OFF period which results in user unfairness. Hence, in this paper, we have considered a scenario where AP can access the medium in both LTE-U ON and OFF periods which results in user unfairness. To study the fairness and channel utilization of Wi-Fi in the presence of LTE-U, we consider the scenario as shown in Fig 1 where an LTE-U eNB and a Wi-Fi AP are deployed 20m apart, each with two users. STA1 and STA2 are two Wi-Fi users with STA1 being a victim user. The interference from LTE-U causes STA1's $S I N R$ to decrease below a minimum required SINR in LTE-U ON period while STA2 is a non-victim user. The path loss $[9]$ in dB between LAA eNB/Wi-Fi AP operating in unlicensed spectrum is

$$
P L=36.7 \log _{10}(d)+22.7+26 \log _{10}(f)
$$

where $d$ is the distance in meters, $f$ is the operating frequency in GHz. The simulation parameters are as shown in Table 1. Figs. 3 and 4 show the SINR variation of Wi-Fi AP in LTE-U OFF and LTE-U ON periods, respectively. In LTE-U OFF period both STAs can transmit and receive as each STA has sufficient SINR, and can be seen from Fig. 3. Whereas from Fig. 4 it is clear that in LTE-U ON period STA-1 can not transmit or receive as it experiences very low SINR $(-0.0006819 \mathrm{~dB})$ whereas STA-2 can still transmit and receive because of its high SINR $(13.45 \mathrm{~dB})$. For this experiment, we considered a full buffer case for IEEE 802.11n based Wi-Fi network, where all Wi-Fi users and the Wi-Fi AP always have data to transmit in UpLink (UL) and DownLink (DL), respectively. For LTE-U network, a similar full buffer case is assumed where unlicensed spectrum of bandwidth $20 \mathrm{MHz}$ is used only for sending DL traffic. LTE-U uses a centralized scheduler while Wi-Fi follows DCF mechanism at MAC layer for sharing radio resources. 


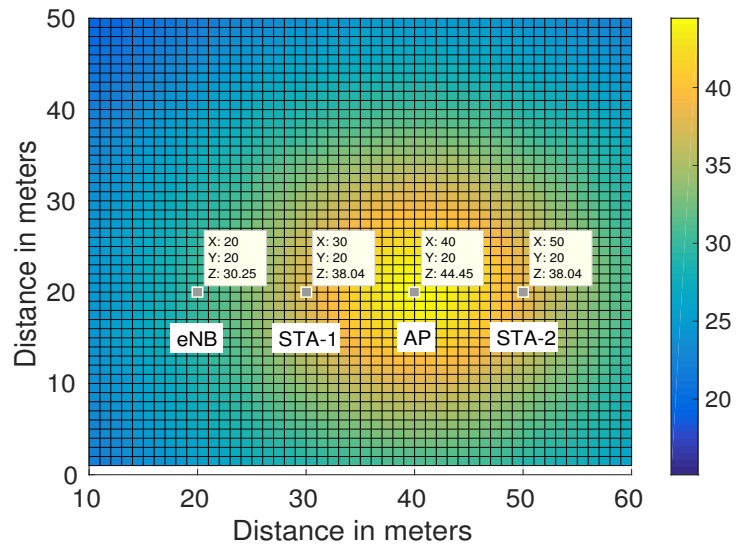

Figure 3: SINR distribution of Wi-Fi AP during LTE-U OFF period. Both Wi-Fi users STA-1 and STA-2 experience good SINR. The coordinates of all the devices are represented with $\mathrm{X}, \mathrm{Y}$-axis with $\mathrm{Z}$-axis as their SINR.

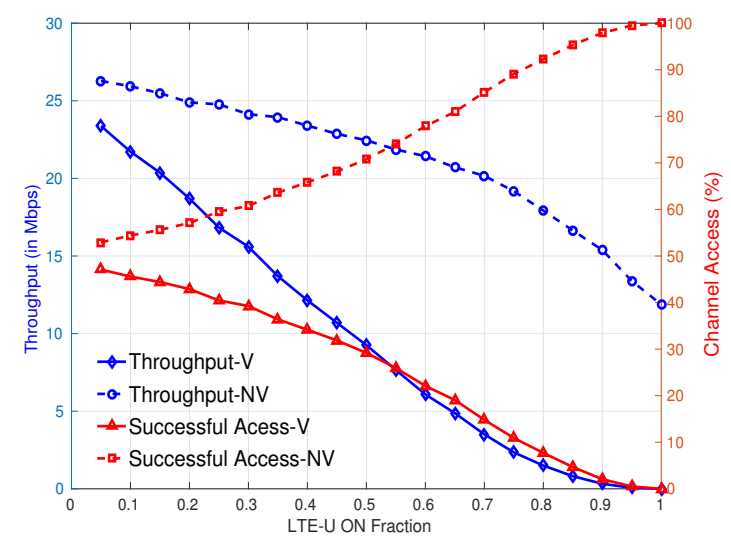

Figure 5: Total Throughput (UL+DL) \& successful channel access (\%) of Wi-Fi users with varying LTE-U ON Fraction $(\eta)$. Victim user (STA1) has inevitably less successful access percentage and throughput as compared to the non-victim user (STA2).

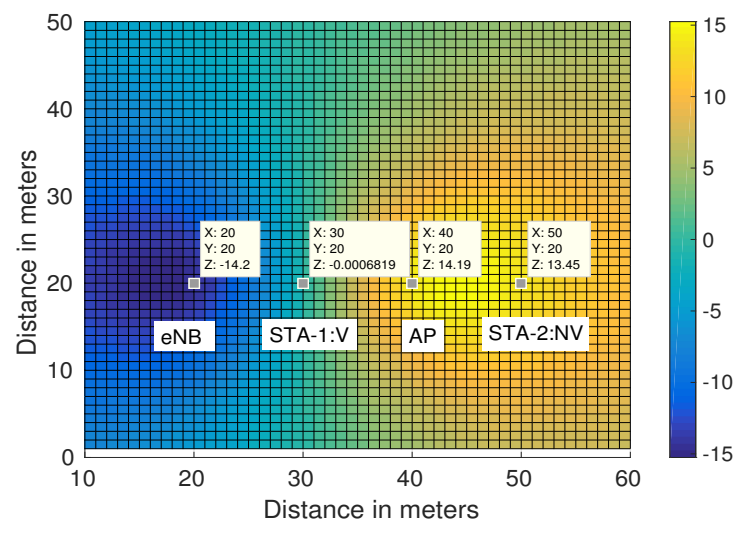

Figure 4: SINR distribution of during LTE-U ON period. STA-1 being placed in between LTE-U eNB and Wi-Fi AP receives very low SINR and becomes a Victim $(\mathrm{V})$ whereas another user (i.e. STA-2) still experiences fairly good SINR, and stays as a Non-Victim (NV).

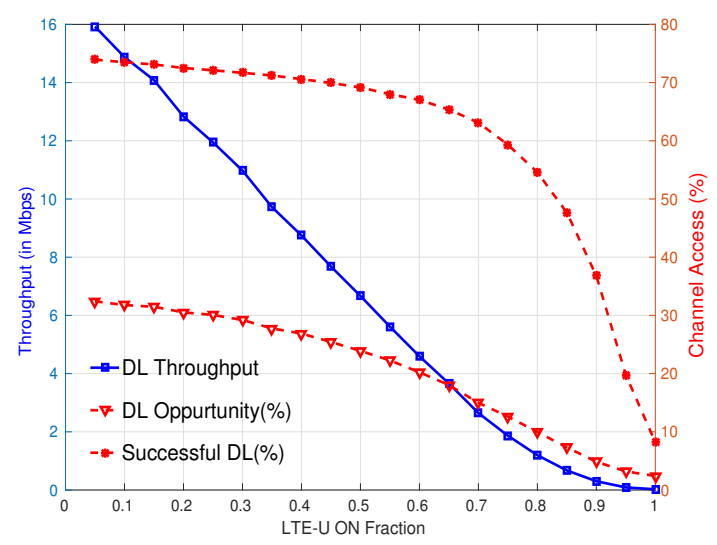

Figure 6: Wi-Fi AP DL performance in UL + DL traffic. Decrease in DL throughput of Wi-Fi AP is the result of decreased DL access opportunities and successful DL percentage.

If LTE-U detects the presence of a Wi-Fi user (by periodically listening to the channel), LTE-U inherently follows an ON-OFF duty cycle 4]. We varied the fraction of time LTE-U is ON $(\eta)$ in a duty cycle period from 0 to 1 and observed the performance of Wi-Fi users, namely the victim and non-victim users.

\section{Key observations from our study are:}




\subsection{Throughput of a non-victim user is always greater than the throughput of a victim user}

During the LTE-U OFF period, both victim and non-victim users will get equal opportunity to access the channel and thereby achieve equal throughputs. But during the LTE-U ON period, the channel can be successfully accessed only by the non-victim users causing the throughput of non-victim users to be higher than the throughput of victim users. Therefore, irrespective of what $\eta$ is, non-victim users will always have higher throughput than victim users.

In principle, LTE-U ceases to access the channel, during its OFF period, so that the victim users not only get channel access but also transmit and receive their packets satisfying minimum SINR requirement. Non-victim users access to the channel - during this (LTE-U OFF) period - engenders unequal, sometimes very low throughput for the victim users. Fig. 5 presents a simulation result consistent with the scenario discussed above, showing the throughput of Wi-Fi users with different $\eta$. It is conspicuous from the figure that the throughput of victim user is less compared to that of non-victim user for all $\eta$.

\subsection{Disproportionate successful channel access by victim and non-victim users}

Fig. 5 shows the successful channel access percentage (defined as the ratio of successful attempts of transmitting or receiving packets by a user to the total successful attempts by all users in the network) of each user with $\eta$. Clearly, the victim user has an inevitably less channel access percentage as compared to the non-victim user which also expounds the fact that the throughput variation is not because of unequal SINR, but rather due to their disproportionate access to the medium. Note that the channel access percentage of a user is the ratio of sum of its UL and DL successful transmissions to the total successful transmissions during the simulation time.

\subsection{High retransmission loss because of victim users}

Packets from the Wi-Fi AP to victim user (STA1) cannot be successfully received, during the LTE-U ON period, due to the low SINR. This results in retransmission of the packets to the victim user multiple times, with every retransmission the Contention Window $(\mathrm{CW})$ at the MAC layer increases exponentially, resulting in increase in average Back Off (BO) value of Wi-Fi AP. This increase reduces the channel access opportunity of the Wi-Fi AP. From Fig. 6, we observe a significant decrease in the DL opportunity percentage (defined as the ratio of Wi-Fi AP DL access opportunities to the total access opportunities in Wi-Fi network) and successful DL percentage (defined as the ratio of successful DL transmissions to the total DL transmissions by AP) with increase in $\eta$. Fig. 6 also shows a decrease in DL throughput of Wi-Fi AP, which is a result of decreased DL access opportunities and successful DL transmissions.

From these observations, it is clear that even if LTE-U shares the channel (by following a duty cycle approach), victim users are severely effected, thereby decreasing the performance of Wi-Fi network. This motivates the need for new solutions to improve the performance of Wi-Fi networks in the presence of LTE-U.

\section{Related Work}

A lot of work is going on, to make LTE operate in unlicensed spectrum by fairly sharing the channel resources with other technologies in unlicensed bands. The benefits and challenges of deploying LTE in unlicensed bands are discussed in 10, 11, 12, 13. LTE is centrally scheduled and always remains ON whereas Wi-Fi uses distributed CSMA/CA, where a device transmits only if the channel is sensed idle. LTE in unlicensed without any modifications in the current LTE Std. will significantly degrade the performance of Wi-Fi, primarily because of the always ON nature of LTE operation, which is clearly delineated in [14, 15, 16]. In [17, the performance of LTE and Wi-Fi in a shared frequency band was presented which again showed that LTE degrades the performance of Wi-Fi, but to improve the performance of Wi-Fi a 
muting technique was introduced within LTE while maintaining fairly good performance of LTE. In 18 and [19, the authors suggested a modified almost blank subframe approach in LTE for fair coexistence with Wi-Fi. Many similar modifications to LTE are further proposed for fair coexistence with Wi-Fi. However, two solutions the Licensed Assisted Access (LAA) and the LTE in unlicensed (LTE-U), have received a lot of attention. The LAA 2 have been a part of 3 GPP standard whereas LTE-U originated from the LTE-U forum [20. Nonetheless, [5] shows both the solutions as equally fair with the Wi-Fi and provides a full authority to the network operator to choose one among them.

A simple approach requiring minimum changes in LTE operation is to follow a discontinuous transmission pattern (a duty cycle pattern) or CSAT where LTE-U follows an ON - OFF cycle 4. Many studies under LTE-U forum 21, 22, 23, demonstrated that the CSAT mechanism allows LTE to be a fair neighbor to Wi-Fi when sharing the same unlicensed channel, by ensuring that the performance of the Wi-Fi AP is no worse than when it shares the same channel with another Wi-Fi AP. However, the duty cycled transmission or CSAT mechanism may be fair with the Wi-Fi network, but it is not fair to all Wi-Fi users as shown in our motivation results. Further for better coexistence of LTE and Wi-Fi, traffic offloading and resource sharing are proposed in 24. The nash bargaining solution is proposed in 25 to develop joint Wi-Fi to LTE-U user transfer and resource allocation strategy. One major challenge which has received very less attention is a scenario where Wi-Fi AP and LTE-U eNB devices use the same channel with Wi-Fi AP being unable to detect the presence of LTE-U eNB as it is outside energy detection range of LTE-U eNB. But, it is not the same with the Wi-Fi clients. Few clients, either because they are in the energy detection range of LTE-U eNB or that the LTE-U transmissions cause interference to such an extent that these clients fail to maintain their minimum SINR requirement, get affected to a large extent. In such scenarios, it lies with the LTE-U eNB either to share or not to share the channel with the Wi-Fi network. If LTE-U eNB does not share the channel, the throughput of such Wi-Fi clients (i.e., victim users) reaches to zero or close to zero. If LTE-U shares the channel by following a duty cycle, the performance of such victim users increases but would still be less than that of the non-victim users. Furthermore, these victim users also degrade the overall Wi-Fi network throughput. This degraded victim users throughput and subsequently the decreased overall network throughput is clearly demonstrated in our motivational example. Hence, existing solutions may degrade the performance of some of the Wi-Fi users in the case of hidden terminal scenario (i.e., with the LTE-U eNB being hidden from Wi-Fi AP). The state-of-art approach to solve hidden/expose terminal problems is to use the Request-to-Send and Clear-to-Send (RTS/CTS) 26] mechanism. But in case of LTE-U and Wi-Fi hidden terminal problem, LTE-U does not understand the RTS/CTS handshake messages of Wi-Fi as both technologies are different from each other. A lot of research has been done on hidden and exposed terminal problems in Wi-Fi and ad hoc networks 27, 28, 29, 30. In literature, inter-Radio Access Technology (RAT) (in this case LTE-U and Wi-Fi) hidden terminal problem has not received much attention. Therefore, in this paper, we propose a solution which not only aims at improving the performance of victim users but also significantly improves the overall Wi-Fi network performance. In addition, the efficacy of the proposed solution is also validated through analysis and simulation results.

\section{System Model}

We consider a scenario where LTE-U eNB and Wi-Fi AP operating on the same unlicensed channel. The users are associated with either LTE-U or Wi-Fi, with LTE-U following a duty cycle based scheme for fair coexistence with Wi-Fi. The fraction of time LTE-U uses for transmission $(\eta)$ in duty cycle period depends on Wi-Fi performance operating on same channel 4 such that performance of Wi-Fi in Wi-Fi-LTE-U coexistence scenario should be equal to or better than Wi-Fi-Wi-Fi coexistence scenario. Though LTE-U is fairly sharing the channel with Wi-Fi by following duty cycled transmission, the mutual interference between Wi-Fi and LTE-U networks remains a major issue for efficient unlicensed spectrum utilization. Hence, to make efficient use of unlicensed spectrum and for better coexistence between LTE-U and Wi$\mathrm{Fi}$, it is important to have some kind of (indirect) coordination between LTE-U and Wi-Fi AP devices. Therefore, we assume that there is an inter-RAT coordinator/controller as in 25, 31, 32. Such inter-RAT 
coordinator/controller can be implemented by the operator if both LTE-U and Wi-Fi AP are deployed by the same operator or it can be implemented by a third party vendor to extend the service to different operators. An alternative to inter-RAT controller can be a logical interface between LTE and Wi-Fi, where Wi-Fi AP shares information regarding beacon interval along with start time of beacon to LTE eNB whereas eNB transfers LTE-U ON fraction information to Wi-Fi AP. Hence, the exchange of information for coordination between LTE-U eNB and Wi-Fi AP can be done with help of logical interface or by using some inter-RAT controller.

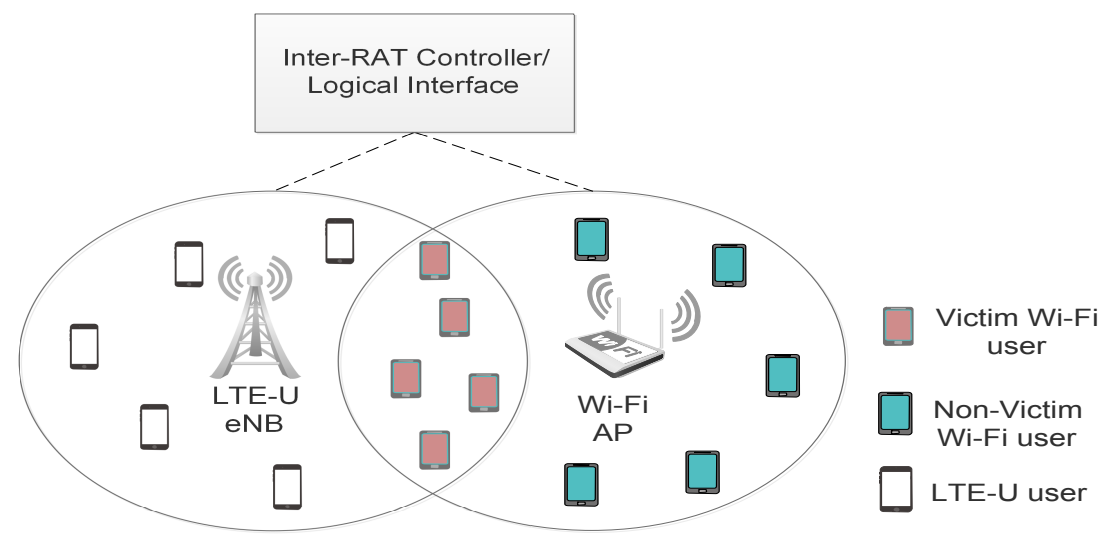

Figure 7: Wi-Fi-LTE-U coexistence system model.

In this work, it is assumed that inter-RAT controller or logical interface can be used to loosely synchronize the LTE-U duty cycle period with the Wi-Fi beacon interval (in multi-eNBs and multi-APs scenario, the controller will synchronize all the Wi-Fi APs and the LTE-U eNBs to a single beacon interval). Beacons in $\mathrm{Wi}-\mathrm{Fi}$ are the management frames, sent by the Wi-Fi AP periodically, containing the network information, and beacon interval is the frequency at which these beacons are sent. After synchronization, if AP changes the beacon interval, it will re-advertise the beacon transmission time and the beacon interval to LTE-eNB via the controller. Now it is made as the responsibility of eNB to match its duty cycle period to the beacon interval (i.e., LTE OFF period + LTE ON period = beacon interval) and always start the OFF period at the beginning of new beacon interval. LTE-U eNB also informs the fraction of time it is ON $(\eta)$ to the AP via the controller and allows Wi-Fi AP to improve channel utilization. Fig. 7] shows the considered system model where LTE-U cell and Wi-Fi BSS are partially overlapped. Whenever Wi-Fi AP has a victim user it starts following coordinated approach. Cellular users are not considered as victim users because LTE eNB can serve them using the licensed spectrum whereas Wi-Fi AP has only unlicensed spectrum to serve its users.

\section{Proposed Work}

Observations from our motivational example persuades one, for a need to adopt a scheme that ensures the fairness among Wi-Fi users. The IEEE 802.11 DCF mechanism during both the LTE-U ON and LTE-U OFF durations, without any intelligent steps to improve the Wi-Fi network performance-despite having throughput knowledge of the users - can be identified as the main bottleneck. Our work identifies this bottleneck, and then proposes an intelligent and controlled use of Point/Hybrid Coordination Function mechanism, without much modification to the existing 802.11 system, to address this issue and thereby harnesses maximum gain. As the proposed scheme is based on Point/Hybrid Coordination Function and it is mainly used in the presence of LTE-U for coexistence hence, we call our proposed scheme as Coexistence Coordination Function (CCF). The CCF mechanism can be used in Wi-Fi networks in the presence of any technology where the device follows the ON-OFF transmissions like LTE-U. 


\subsection{Operation of Coexistence Coordination Function (CCF)}

A Point Coordination Function (PCF) 33 is a poll and response protocol used in Wi-Fi to eliminate the possibility of contention among users for the medium. It has a Point Coordinator (PC) located inside Wi-Fi AP which controls the PCF. In PCF, the channel is divided into two periods-Contention Free Period (CFP) and Contention Period (CP). AP sends beacon frame after which CFP starts, a Contention-Free(CF)-poll (CF-poll) frame is sent by AP to users to permit transmission. A CF-end frame is transmitted by AP to stop CFP and begin CP. An enhancement to PCF in Wi-Fi for Quality of Service (QoS) is the contention free channel access protocol called Hybrid Coordination Function controlled channel access (HCCA) 26] where Hybrid Coordination Function (HCF) similar to PCF controls the iteration of CFP and CP. A major difference between $\mathrm{PCF}$ and $\mathrm{HCF}$ is that in PCF, CF-polling can be issued only in CFP whereas, in HCF a CF-polling can be issued in both $\mathrm{CFP}$ and $\mathrm{CP}$. The proposed scheme explained below uses the CFP and $\mathrm{CP}$ of Wi-Fi effectively to achieve fairness among Wi-Fi users in terms of throughput and successful channel access, and at the same time, improves the overall performance of Wi-Fi network.

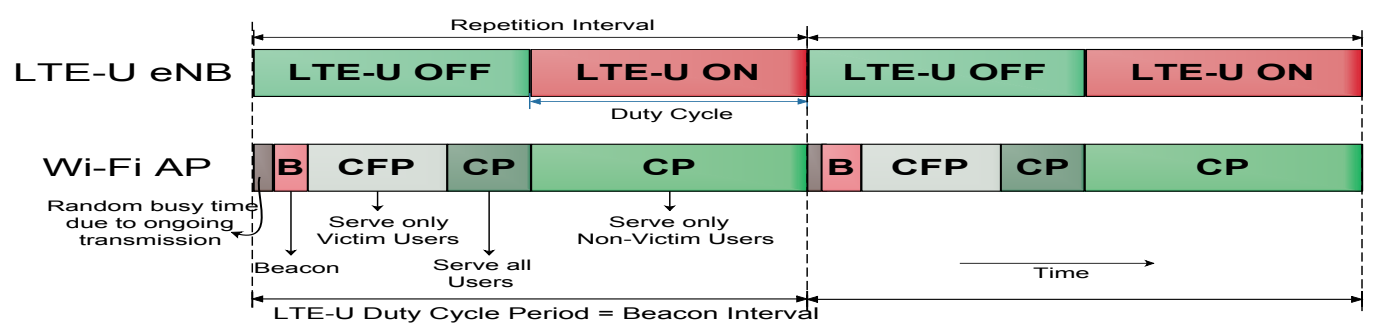

Figure 8: CCF mechanism for Wi-Fi in the presence of LTE-U eNB.

The scenario presented in the system model requires a control in the channel access of non-victim users accompanied by a special channel access given to the victim users. To accomplish the above goal, we advocate usage of $\mathrm{PCF} / \mathrm{HCF}$ mode of $\mathrm{Wi}-\mathrm{Fi}$ [26], where the channel has two periods as $\mathrm{CFP}$ and $\mathrm{CP}$; with the difference being that the $\mathrm{CP}$ involves contention among users whereas CFP requires only those users to be served which are polled or selected by the Wi-Fi AP. Since victim users cannot be served in the LTE-U ON period; our CCF mechanism as shown in Fig. 8 allows the Wi-Fi AP to transmit and receive data from victim users during the LTE-U OFF period-by using CFP. After the victim users are served, for the remaining LTE-U OFF period, AP follows $\mathrm{CP}$ (i.e., DCF) to serve all the users, with each user contending and gaining access to the medium. With the onset of LTE-U ON period, Wi-Fi network still continues to be in $\mathrm{CP}$, but Wi-Fi AP having the additional knowledge of victim users, does not attempt to serve the victim users. This prevents unnecessary re-transmissions and helps the Wi-Fi AP to maintain its channel access opportunity same as non-victim users. The complete operation is illustrated in Fig. 8, Also, the loose synchronization of beacon interval with LTE-U duty cycle period helps Wi-Fi AP to send its beacon at the beginning of LTE-U OFF period. This ensures that every Wi-Fi user listens to them, which is important for a user to maintain its association with the Wi-Fi network.

\subsection{Optimum value of CFP in CCF mechanism}

If the entire LTE-U OFF period is used to serve the victim users (by making entire LTE-U OFF duration as CFP), the result can be, victim users gaining undue advantage in terms of throughput. To avoid this, we find the minimum CFP duration, within the LTE-U OFF period, that is required for ensuring throughput fairness among the Wi-Fi users. If $x$ is the duration to operate in $\mathrm{CFP}$, variation in $x$ can give control of how much the victim users has perquisite over the non-victim. When $x$ is made to zero, the CCF degenerates to DCF mechanism. Any value of $x$ between 0 and 1- $\eta$ will give better throughput for the victim users (if present) as well for the entire Wi-Fi setup. One approach to realize minimum CFP duration is to adjust $x$ 
by observing the throughputs of users in the previous duty cycle period. The updated CFP duration $\left(T_{c f p}\right)$ in terms of previous CFP duration $T_{c f p}^{o l d}$ can be given as

$$
T_{c f p}=\min \left(\frac{\Gamma_{n v}^{n e w}}{\Gamma_{v}^{n e w}} \cdot T_{c f p}^{o l d}, \text { LTE-U_OFF_Duration }\right)
$$

$\Gamma_{n v}^{n e w}$ and $\Gamma_{v}^{n e w}$ are calculated using weighted moving average throughputs of non-victim and victim users, respectively as

$$
\begin{aligned}
& \Gamma_{n v}^{n e w}=(1-\alpha) \Gamma_{n v}^{\text {prev }}+\alpha \Gamma_{n v}^{o l d} \\
& \Gamma_{v}^{\text {new }}=(1-\alpha) \Gamma_{v}^{\text {prev }}+\alpha \Gamma_{v}^{o l d}
\end{aligned}
$$

Where $\Gamma_{n v}^{\text {prev }}$ and $\Gamma_{v}^{p r e v}$ are average throughputs in the previous duty cycle period and $\Gamma_{n v}^{o l d}$ and $\Gamma_{v}^{o l d}$ are the $\Gamma_{n v}^{n e w}$ and $\Gamma_{v}^{n e w}$ of the previous duty cycle period, for non-victim and victim user, respectively and $\alpha$ is a smoothing parameter.

As observed in the Section 2, the victim user is deprived of packets in LTE-U ON period whereas nonvictim user receives them in both the LTE-U ON and OFF periods. Hence, the throughput of non-victim user elevates as compared to that of the victim user. Our goal is to achieve fairness by giving some duration to victim users (i.e., $T_{c f p}$ ) to ensure comparable throughputs to the victim and non-victim users. Hence, we define the fairness in terms of average throughput of victim and non-victim users using Jains fairness index 34$]$.

$$
\text { fairness }=\frac{\left(\Gamma_{v}+\Gamma_{n v}\right)^{2}}{2 \times\left(\left(\Gamma_{v}\right)^{2}+\left(\Gamma_{n v}\right)^{2}\right)}
$$

where $\Gamma_{v}$ and $\Gamma_{n v}$ are average throughputs of victim and non-victim users, respectively.

\subsection{Identification of victim and non-victim users by Wi-Fi AP in CCF mechanism}

The presence of LTE-U eNB divides the Wi-Fi users into two groups - victim and non-victim users as shown in the motivational example in Fig. 1. Hence, Wi-Fi AP needs to differentiate between victim and non-victim users to serve them efficiently. In our proposed mechanism, a Wi-Fi user informs to the AP whenever it detects any non-Wi-Fi signal, with its capability to differentiate Wi-Fi and non-Wi-Fi signals using Wi-Fi preamble. Though, apart from LTE-U, Radar signals may also be present in the same $5 \mathrm{GHz}$ spectrum, but a Wi-Fi network normally shifts from the radar channel, avoiding the possibility of these nonWi-Fi signals to be Radar signals. Hence non-Wi-Fi signals are only due to the LTE-U transmissions. And if these non-Wi-Fi signals cause interference to an extent that Wi-Fi users minimum SINR requirement is not satisfied, to establish a successful communication, such users are classified as victim users. Algorithm 1 helps to identify the victim and non-victim users, while running in the background at Wi-Fi AP. In essence, those users, who succumb to lower or zero throughputs in LTE-U ON period but not in LTE-U OFF period of LTE-U, due to the presence of an LTE-U eNB, are considered as victim users and other as non-victim users. Whenever Wi-Fi AP has a victim user it starts following a coordination approach that we discuss in the following section.

\subsection{Workflow of the CCF mechanism}

Fig. 9 shows the data flow diagram of our CCF mechanism with a high-level description for each step involved.

In Step 1, AP initiates a coordinated approach if AP has any victim users. Here, the AP informs the beacon interval and the expected start time of beacon (as the actual transmission time of beacon may get shifted because of a busy medium, the expected time as opposed to the actual time is informed). In Step 2, 

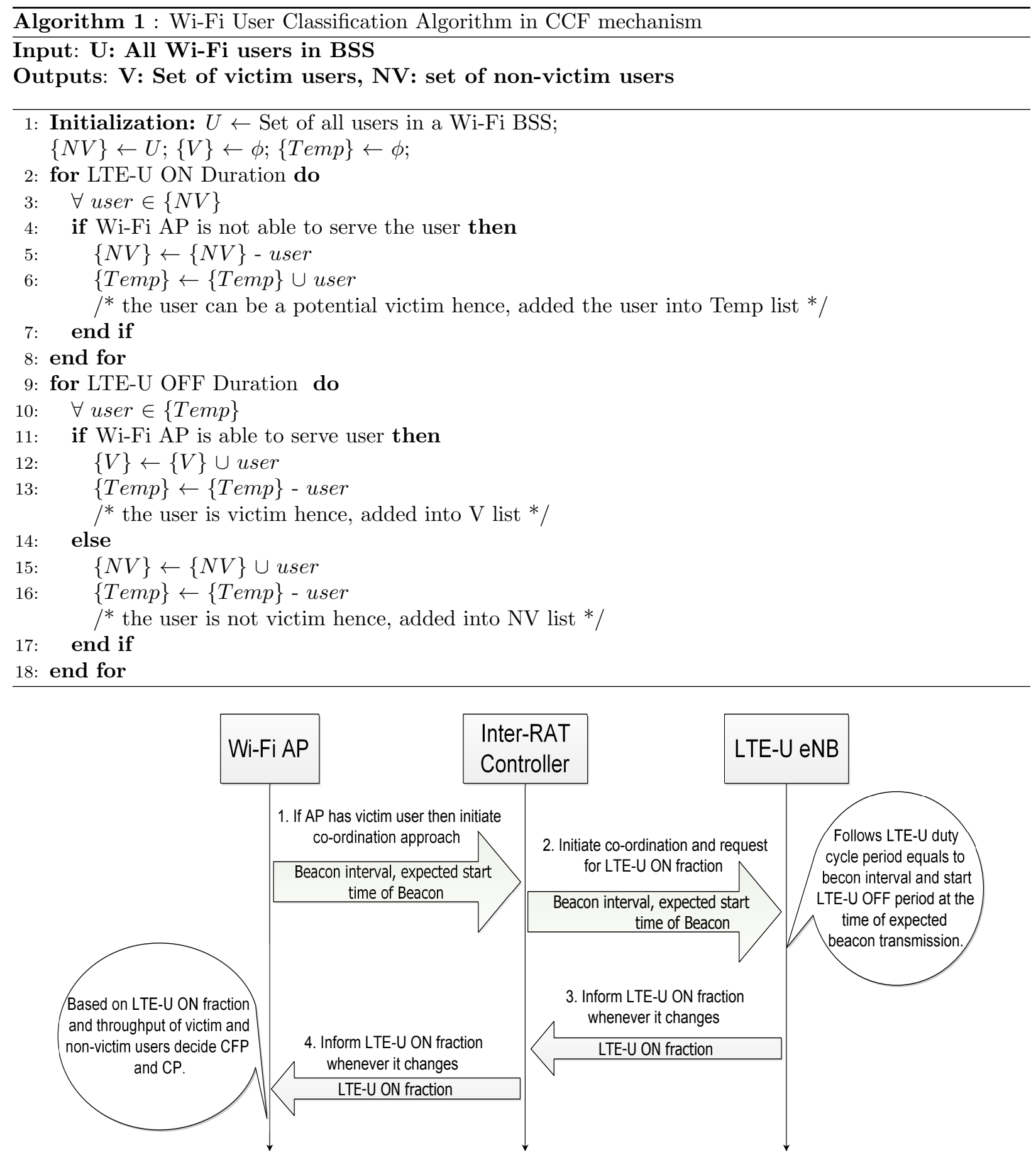

Figure 9: Data flow diagram for the CCF with inter-RAT controller.

the Inter-RAT controller forwards the beacon interval and expected start time of beacon to the LTE-U eNB, and requests the eNB to send LTE-U ON fraction.

After knowing beacon interval and expected start time of beacon, LTE-U eNB sets its duty cycle period 
equal to the beacon interval and starts the LTE-U OFF period at the expected start time of beacon transmission. Hence, after every beacon interval, the eNB starts its LTE-U OFF period at the time of new a beacon transmission. However, it does not matter whether the beacon is transmitted by AP exactly at the time of beacon transmission or after some delay because of the medium being busy. In this way, LTE-U eNB will only be loosely synchronized with Wi-Fi AP. The eNB will always start its LTE-U OFF period at the predefined time of beacon transmission which is calculated based on the beacon interval and the previous transmission time of beacon.

In Step 3, LTE-U eNB informs the LTE-U ON fraction to the controller. Henceforth whenever eNB changes its LTE-U ON fraction it informs the same to the controller. In Step 4, the controller forwards the LTE-U ON fraction to AP. Similarly, whenever LTE-U changes its LTE-U ON fraction, the controller forwards this new ON fraction to the AP.

After knowing the LTE-U ON fraction Wi-Fi AP calculates CFP duration using Eqn. (1) and serves the victim users in CFP. If LTE-U is still in OFF period, the Wi-Fi AP now serves all of its users in CP. Finally, in LTE-U ON period where Wi-Fi already serving in CP, serves only the non-victim users.

\section{Throughput Analysis of DCF and CCF mechanisms}

For analyzing throughput of CCF mechanism, we consider an LTE-U eNB and a Wi-Fi AP, each having $N_{t}$ clients. Wi-Fi throughput with $\mathrm{PCF} / \mathrm{HCF}$ mode enabled (CCF mechanism) differs extensively from its standard mode of operation (i.e., a Wi-Fi network with nodes configured only in DCF mode). We compare the throughput of CCF mechanism (Proposed Scheme) with that of the DCF mechanism (Standard Wi-Fi). LTE-U is assumed to serve its unlicensed users in DL 2], and Wi-Fi is analyzed for saturated DL only traffic.

Let $T_{\text {difs }}, T_{\text {slot }}$, and $T_{\text {sifs }}$ denote the DIFS interval, back-off slot time, and SIFS interval for the Wi-Fi network, respectively. Let $T_{b u s y}$ denote the amount of time channel will be busy after a data transmission start. Therefore, $T_{\text {busy }}=T_{\text {rts }}+T_{\text {sifs }}+T_{c t s}+T_{\text {sifs }}+T_{\text {data }}+T_{\text {sifs }}+T_{\text {ack }}$, where $T_{\text {data }}$ represents the average time required to successfully transmit a data packet - when LTE-U is ON and $T_{a c k}$ represents the average time required to transmit an ACK. Let $Q_{K}$ denote the random back-off value selected by the Wi-Fi AP during the $K^{\text {th }}$ transmission and $T_{\text {succ }}$ denotes the average time taken by the AP to transmit one data burst successfully. Since a DL only traffic scenario will not elicit any collisions, $P\left(Q_{K}=q_{k}\right)=\frac{1}{W}$, where $\mathrm{W}$ denotes the minimum back-off window size and average value of $q_{k}$ is given as

$$
E\left[q_{k}\right]=\frac{W-1}{2}
$$

and then the expression for $T_{\text {succ }}$ is given by

$$
\begin{aligned}
T_{\text {succ }} & =E\left[T_{\text {difs }}+T_{\text {slot }}\left(q_{k}\right)+T_{\text {busy }}\right] \\
& =T_{\text {difs }}+\left(\frac{W-1}{2}\right) T_{\text {slot }}+T_{\text {busy }}
\end{aligned}
$$

To determine the throughput of such scenario, we employ slot based approach, which assumes that Wi-Fi AP follows a process of transmitting the data on a per slot basis. A slot in essence, is determined by the time it takes to transmit to a victim or a non-victim user. During the LTE-ON period, successful transmission to a victim user is indeed not possible (due to low SINR). Hence, the Wi-Fi AP will keep on sending Request-to-Send (RTS) packets, until the number of attempts reaches the maximum retry limit $\left(R_{\text {limit }}\right)$, before it discards the packet. Nonetheless, a non-victim user can be served successfully during this period (assuming an ideal channel). Therefore, on an average a successful transmission will last for 
$T_{\text {succ }}$ and an unsuccessful, involving Clear-to-Send (CTS) time out duration $\left(T_{\text {cts_timeout }}\right), R_{\text {limit }}$ number of re-transmissions and exponential growth of $\mathrm{CW}$ with every re-transmission, will last for $T_{u n-s u c c}$, where

$$
T_{\text {un-succ }}=\frac{1}{2}\left[2^{m} W\left(R_{\text {limit }}-m+1\right)-R_{\text {limit }}-W\right] T_{\text {slot }}+R_{\text {limit }}\left(T_{\text {difs }}+T_{\text {rts }}+T_{\text {cts_timeOut }}\right)
$$

and, $m$ denotes $\log _{2}\left(\frac{C W_{\max }}{W}\right)$. Let $T_{i}$ be a random variable representing the time consumed by any slot, which is given by

$$
\begin{aligned}
& T_{i}=\left\{\begin{array}{cc}
T_{\text {un-succ }} & \text { with prob. } p \\
T_{\text {succ }} & \text { with prob. } 1-p
\end{array}\right\} \\
& \text { and } E\left[T_{i}\right]=p T_{\text {un-succ }}+(1-p) T_{\text {succ }}
\end{aligned}
$$

where $p=\frac{N_{v}}{N_{t}}$, assuming that AP randomly selects a user to serve, with no special preferences.

The total time taken by $n$ such slots will be given by

$$
T=\sum_{i=1}^{n} T_{i} \Longrightarrow E[T]=E\left[\sum_{i=1}^{n} T_{i}\right]=\sum_{i=1}^{n} E\left[T_{i}\right]
$$

Since, $T_{i}^{\prime} s$ are Independent and Identically Distributed (IID) copies of each other,

$$
E[T]=n E\left[T_{i}\right]
$$

Using $T_{\text {succ }}$ and $T_{\text {un-succ }}$, we categorize the performance of DCF and CCF mechanisms to show the benefits in terms of fairness achieved among Wi-Fi users and throughput improvement in Wi-Fi network.

\subsection{Throughput analysis of DCF mechanism}

The complete operation of DCF mechanism in the OFF and ON periods is shown in Fig. 10. Transmissions to both victim and non-victim users are shown as successful in the LTE-U OFF period. But, in LTE-U ON period, the transmission to victim users would result in a packet loss. Moreover, when AP tries to retransmit the same packet, it further results in multiple retransmission of the same packet to the victim user. However, the transmissions to a non-victim user are still successful in the ON period.

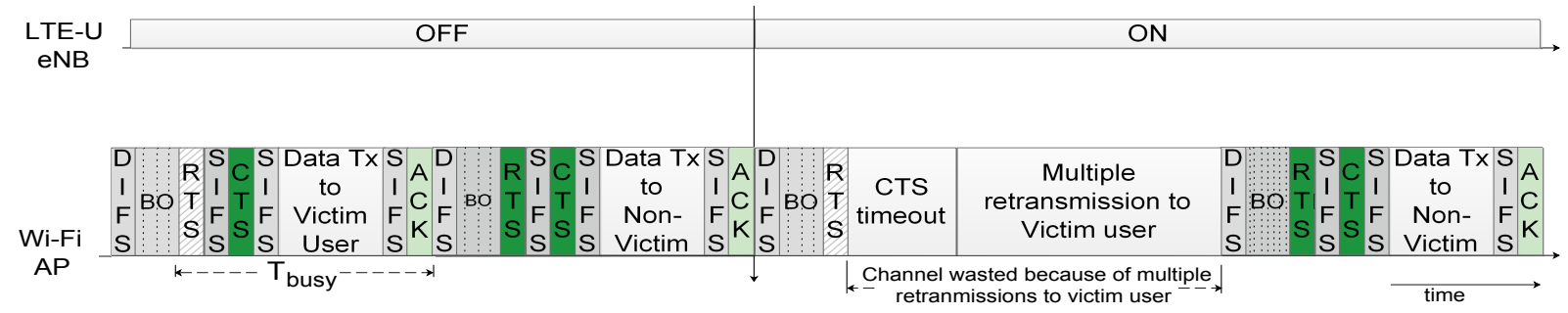

Figure 10: Operation of DCF mechanism for victim and non-victim users in duty cycled LTE-U.

Since the behavior of Wi-Fi during the LTE-ON and LTE-OFF periods is quite dissimilar, we analyze both of them separately. During the LTE-OFF period, all the Wi-Fi transmissions are successful, implying that there is no randomness involved in the slot duration $\left(T_{i}\right)$.

$$
T_{i}=T_{\text {succ }}^{\prime} \quad \text { and } \quad E[T]=n T_{\text {succ }}^{\prime}
$$


$T_{\text {succ }}^{\prime}$ differs from $T_{\text {succ }}$ in $T_{\text {data }}$; no interference from LTE-U causes Wi-Fi AP to use higher Modulation and Coding Scheme (MCS) and thus will decrease $T_{\text {data }}$, and $T_{\text {succ }}^{\prime}<T_{\text {succ }}$. If average size of packet is $E[P]$ (bytes), then the total number of bytes transmitted during this interval will be $n E[P]$ and the throughput of network during the LTE-OFF period $\left(\Gamma_{o f f}^{D C F}\right)$ will be

$$
\Gamma_{o f f}^{D C F}=\frac{n E[P]}{n T_{\text {succ }}^{\prime}}=\frac{E[P]}{T_{\text {succ }}^{\prime}}
$$

The throughput of victim user $\left(\Gamma_{o f f, v}^{D C F}\right)$ and non-victim user $\left(\Gamma_{o f f, n v}^{D C F}\right)$ will be same and are given by

$$
\Gamma_{o f f, v}^{D C F}=\Gamma_{o f f, n v}^{D C F}=\frac{\Gamma_{o f f}^{D C F}}{N_{t}}=\frac{1}{N_{t}} \frac{E[P]}{T_{s u c c}^{\prime}}
$$

During the LTE-ON period, AP will be able to successfully transmit to a non-victim user, but its transmission to a victim user, will be unsuccessful. This will cause $T_{i}$ to be a random variable, fluctuating between $T_{\text {succ }}$ and $T_{u n-s u c c}$, given by Eqn (6) and Eqn (7). The average duration to complete $n$ transmissions $(E[T])$ during this interval is given by

$$
E[T]=n E\left[T_{i}\right]=n\left(p T_{\text {un-succ }}+(1-p) T_{\text {succ }}\right)
$$

Data packet is transmitted only when a successful transmission takes place, i.e., if the packet is meant for a non-victim user, implying that the average amount of data successfully transmitted is the sum of all transmissions to the non-victim users in the Wi-Fi network. Therefore, the average amount of data transmitted is $n(1-p) E[P]$. The total throughput $\Gamma_{o n}^{D C F}$ can then be given by

$$
\Gamma_{o n}^{D C F}=\frac{(1-p) E[P]}{\left(p T_{u n-s u c c}+(1-p) T_{\text {succ }}\right)}
$$

Since the victim users do not receive any data, their throughputs $\left(\Gamma_{o n, v}^{D C F}\right)$ will be zero $\left(\Gamma_{o n, v}^{D C F}=0\right)$, while the non-victim users will equally share the total throughput as

$$
\Gamma_{o n, n v}^{D C F}=\frac{1}{N_{t}-N_{v}} \frac{(1-p) E[P]}{\left(p T_{\text {un-succ }}+(1-p) T_{\text {succ }}\right)}
$$

The total throughput of a victim user $\left(\Gamma_{v}^{D C F}\right)$ and that of a non-victim user $\left(\Gamma_{n v}^{D C F}\right)$ in a duty cycle period are given by

$$
\begin{gathered}
\Gamma_{v}^{D C F}=(\eta) \Gamma_{o n, v}^{D C F}+(1-\eta) \Gamma_{o f f, v}^{D C F}=(1-\eta) \frac{1}{N_{t}} \frac{E[P]}{T_{\text {succ }}^{\prime}} \\
\text { Similarly, } \Gamma_{n v}^{D C F}=(\eta) \Gamma_{o n, n v}^{D C F}+(1-\eta) \frac{1}{N_{t}} \frac{E[P]}{T_{\text {succ }}^{\prime}}
\end{gathered}
$$

Hence, the total Wi-Fi throughput $\left(\Gamma^{D C F}\right)$ of DCF mechanism network in the presence of LTE-U is

$$
\begin{aligned}
\Gamma^{D C F} & =\left(N_{v}\right) \Gamma_{v}^{D C F}+\left(N_{t}-N_{v}\right) \Gamma_{n v}^{D C F} \\
& =\left(N_{v}\right)\left((1-\eta) \frac{1}{N_{t}} \frac{E[P]}{T_{\text {succ }}^{\prime}}\right)+\left(N_{t}-N_{v}\right)\left((\eta) \Gamma_{\text {on }, n v}^{D C F}+(1-\eta) \frac{1}{N_{t}} \frac{E[P]}{T_{\text {succ }}^{\prime}}\right) \\
& =(\eta)\left(N_{t}-N_{v}\right)\left(\Gamma_{\text {on }, n v}^{D C F}\right)+\left((1-\eta) \frac{E[P]}{T_{\text {succ }}^{\prime}}\right)
\end{aligned}
$$




\subsection{Throughput analysis of CCF mechanism}

The complete operation of Wi-Fi with CCF mechanism in OFF and ON periods of LTE-U is shown in Fig. 11, where Wi-Fi AP serves only victim users in CFP during LTE-U OFF period and only non-victim users in the LTE-U ON period.

Using the information, regarding the presence of victim users and LTE-U ON period, in our CCF mechanism (for efficient utilization of unlicensed spectrum) the Wi-Fi AP intelligently defers from transmitting to the victim users during LTE-U ON period, and serves these users using PCF/HCF mode during LTE-U OFF period. Since LTE-ON period involves only the non-victim users transmissions, every transmission will be successful and will last for $T_{\text {succ }}$ duration. Hence, the throughputs of each non-victim $\left(\Gamma_{o n, n v}^{C C F}\right)$ and victim $\left(\Gamma_{o n, v}^{C C F}\right)$ users are given by

$$
\Gamma_{o n, v}^{C C F}=0 \quad \text { and } \quad \Gamma_{o n, n v}^{C C F}=\frac{1}{N_{t}-N_{v}} \frac{E[P]}{T_{s u c c}}
$$

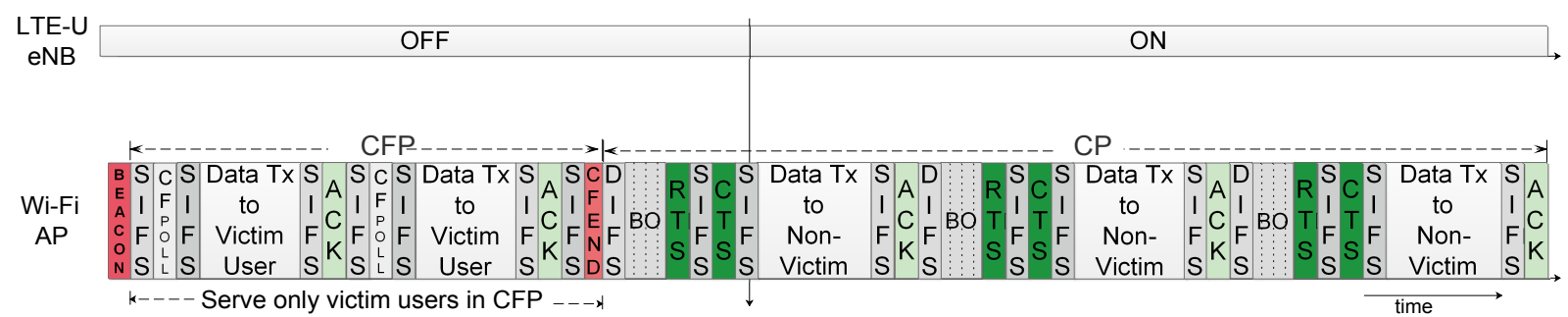

Figure 11: Operation of CCF mechanism for victim and non-victim users in duty cycled LTE-U.

But, during the LTE-OFF period, the behavior of CCF mechanism is quite ingenious. Let $T_{\text {succ }}^{p}$ denote the average time taken to serve a victim user, in LTE-OFF period using CFP. In CFP, AP sends a CF-poll frame to permit transmission to victim users. Let $T_{\text {cfpoll }}$ be the time required for CF-poll frame to transmit then the time taken to transmit a data packet (and receive its ACK) using CFP is given by

$$
T_{\text {succ }}^{p}=T_{\text {sifs }}+T_{\text {cfpoll }}+T_{\text {sifs }}+T_{\text {data }}+T_{\text {sifs }}+T_{a c k}
$$

Assuming average size of packet as $E[P]$, time taken for $n$ such transmissions and the total throughput of Wi-Fi network $\left(\Gamma_{o f f}^{c f p}\right)$ are given by

$$
\sum_{i=1}^{n} T_{\text {succ }}^{p}=n T_{\text {succ }}^{p} \quad \text { and } \quad \Gamma_{\text {off }}^{c f p}=\frac{E[P]}{T_{\text {succ }}^{p}}
$$

As none of the non-victim users are being served in the CFP, their throughputs $\left(\Gamma_{o f f, n v}^{c f p}\right)$ will be zero, while the victim users $\left(\Gamma_{o f f, v}^{c f p}\right)$ equally share the total network throughput as

$$
\Gamma_{o f f, n v}^{c f p}=0 \quad \text { and } \quad \Gamma_{o f f, v}^{c f p}=\frac{1}{N_{v}} \frac{E[P]}{T_{s u c c}^{p}}
$$

With the remaining time in which the LTE is still OFF, Wi-Fi uses PCF mode in CP to serve all the users, which will have the throughput, same as that of DCF mechanism operating in LTE-OFF period $\left(\Gamma_{o f f}^{D C F}\right)$. Hence, the Wi-Fi throughput during the LTE-OFF period within $\mathrm{CP}\left(\Gamma_{o f f}^{c p}\right)$ is given as

$$
\Gamma_{o f f}^{c p}=\Gamma_{o f f}^{D C F}
$$


Also, each victim $\left(\Gamma_{o f f, v}^{c p}\right)$ and non-victim users $\left(\Gamma_{o f f, n v}^{c p}\right)$ will have equal throughputs as

$$
\Gamma_{o f f, v}^{c p}=\Gamma_{o f f, n v}^{c p}=\frac{\Gamma_{o f f}^{D C F}}{N_{t}}
$$

Assuming that the fraction of time, Wi-Fi operates in CFP, during the LTE-U OFF period, as $x \leq(1-\eta)$, the total average throughput of each victim $\left(\Gamma_{v}^{C C F}\right)$ and non-victim user $\left(\Gamma_{n v}^{C C F}\right)$, with the CCF mechanism can be given as

$$
\begin{aligned}
\Gamma_{v}^{C C F} & =(\eta) \Gamma_{o n, v}^{C C F}+(1-\eta-x) \Gamma_{o f f, v}^{c p}+(x) \Gamma_{o f f, v}^{c f p} \\
& =(1-\eta-x) \frac{1}{N_{t}} \frac{E[P]}{T_{\text {succ }}^{\prime}}+(x) \frac{1}{N_{v}} \frac{E[P]}{T_{\text {succ }}^{p}}
\end{aligned}
$$

Similarly,

$$
\begin{aligned}
\Gamma_{n v}^{C C F} & =(\eta) \Gamma_{o n, n v}^{C C F}+(1-\eta-x) \Gamma_{o f f, n v}^{c p}+(x) \Gamma_{o f f, n v}^{c f p} \\
& =(\eta) \frac{1}{N_{t}-N_{v}} \frac{E[P]}{T_{\text {succ }}}+(1-\eta-x) \frac{1}{N_{t}} \frac{E[P]}{T_{\text {succ }}^{\prime}}
\end{aligned}
$$

Hence, the total Wi-Fi throughput $\left(\Gamma^{C C F}\right)$ in our CCF mechanism is given by

$$
\begin{aligned}
\Gamma^{C C F} & =\left(N_{v}\right) \Gamma_{v}^{C C F}+\left(N_{t}-N_{v}\right) \Gamma_{n v}^{C C F} \\
& =\left(N_{v}\right)\left((1-\eta-x) \frac{1}{N_{t}} \frac{E[P]}{T_{\text {succ }}^{\prime}}+(x) \frac{1}{N_{v}} \frac{E[P]}{T_{s u c c}^{p}}\right)+\left(N_{t}-N_{v}\right)\left((\eta) \frac{1}{N_{t}-N_{v}} \frac{E[P]}{T_{\text {succ }}}+(1-\eta-x) \frac{1}{N_{t}} \frac{E[P]}{T_{\text {succ }}^{\prime}}\right) \\
& =(\eta) \frac{E[P]}{T_{\text {succ }}}+(1-\eta-x) \frac{E[P]}{T_{\text {succ }}^{\prime}}+(x) \frac{E[P]}{T_{\text {succ }}^{p}}
\end{aligned}
$$

\subsection{Observations and gains}

(i) Throughput of each victim user will increase irrespective of $\eta$ : Using Eqn (16) and Eqn $25, \Gamma_{v}^{C C F}$ can be re-written as

$$
\Gamma_{v}^{C C F}=\Gamma_{v}^{D C F}+(x)\left(\frac{E[P]}{N_{v} T_{\text {succ }}^{p}}-\frac{E[P]}{N_{t} T_{\text {succ }}^{\prime}}\right)
$$

The second term on the Right Hand Side (RHS) of Eqn (28) indicates whether the CCF mechanism can outperform DCF mechanism in terms of throughput of victim user. The two fractions within the second term are the throughputs of victim user in the $x$ duration with the CCF and DCF mechanisms, respectively. This indicates that the CCF mechanism outperforms DCF mechanism during the duration in which the victim users are served in CFP of PCF mode, but loses some throughput which could be served in the same duration, by DCF mechanism, using CP of DCF mode. Since, $T_{\text {succ }}^{p}<T_{\text {succ }}^{\prime}$ and $N_{v} \leq N_{t}$,

$$
\frac{1}{N_{v}} \frac{E[P]}{T_{\text {succ }}^{p}}>\frac{1}{N_{t}} \frac{E[P]}{T_{\text {succ }}^{\prime}} \Longrightarrow \Gamma_{v}^{C C F}>\Gamma_{v}^{D C F}
$$

(ii) Total throughput of Wi-Fi network will increase regardless of $\eta$ : Using Eqns (18) and $(27), \Gamma^{C C F}$ can be written as

$$
\Gamma^{C C F}=\Gamma^{D C F}+x\left(\frac{E[P]}{T_{s u c c}^{p}}-\frac{E[P]}{T_{\text {succ }}^{\prime}}\right)+\eta\left(\frac{E[P]}{T_{\text {succ }}}-\frac{E[P]}{\left(\frac{p}{1-p} T_{u n-s u c c}+T_{\text {succ }}\right)}\right)
$$

Since, $T_{u n-s u c c}>0$ and $T_{\text {succ }}^{\prime}>T_{\text {succ }}^{p}$, it implies that all the terms on the RHS are positive. Therefore, $\Gamma^{C C F}>\Gamma^{D C F}$ (i.e., throughput of CCF mechanism is greater than that of DCF mechanism). 


\subsection{Maximum value of LTE-U ON Fraction for which fairness is achievable}

The maximum value of LTE-U ON Fraction for which fairness is achievable is based on the insufficiency in LTE-U OFF fraction to maintain the throughput fairness between the victim and non-victim users. The value of $\eta$ at this point is denoted by $\eta_{t}$ and is defined as the maximum value of $\eta$ for which equal average per user throughput between victim and non-victim users is achievable.

The significance of $\eta_{t}$ comes along when LTE-U wishes to increase its ON Fraction. The above $\eta_{t}$ provides an upper bound to this increase to ensure that the fairness is achievable among the Wi-Fi users. Further increase of $\eta$ (i.e., LTE-U ON Fraction) may manifests into an improvement in LTE-U throughput, but on the compromise of destroying the innate behaviour of Wi-Fi to be fair.

The value of $\eta_{t}$ can be obtained by maximizing $\eta$ over the following constraints:

$$
\begin{array}{ll}
\eta \leq 1 & \text { (i.e., The value of LTE-U ON Fraction } \leq 1) \\
\Gamma_{v}^{C C F}=\Gamma_{n v}^{C C F} & \text { (i.e., The throughput of a victim user is equal to that of a non-victim user) }
\end{array}
$$

By equating the throughputs given in Eqns. 25 and 26, we get the following equation,

$$
\begin{aligned}
& (1-\eta-x) \frac{1}{N_{t}} \frac{E[P]}{T_{\text {succ }}^{\prime}}+(x) \frac{1}{N_{v}} \frac{E[P]}{T_{\text {succ }}^{p}}=(\eta) \frac{1}{N_{t}-N_{v}} \frac{E[P]}{T_{\text {succ }}}+(1-\eta-x) \frac{1}{N_{t}} \frac{E[P]}{T_{\text {succ }}^{\prime}} \\
& \Longrightarrow(x) \frac{1}{N_{v}} \frac{E[P]}{T_{\text {succ }}^{p}}=(\eta) \frac{1}{N_{t}-N_{v}} \frac{E[P]}{T_{\text {succ }}} \Longrightarrow x=\eta \frac{N_{v}}{N_{t}-N_{v}} \frac{T_{\text {succ }}^{p}}{T_{\text {succ }}}
\end{aligned}
$$

Now, we use the fact that $x \leq 1-\eta$, which comes along with the definition of $x$, as $x$ is the CFP Fraction within the LTE-U OFF Fraction $(1-\eta)$.

$$
x \leq 1-\eta \Longrightarrow \eta \frac{N_{v}}{N_{t}-N_{v}} \frac{T_{\text {succ }}^{p}}{T_{\text {succ }}} \leq 1-\eta \Longrightarrow \eta \leq \frac{1}{\frac{N_{v}}{N_{t}-N_{v}} \frac{T_{\text {succ }}^{p}}{T_{\text {succ }}}+1}
$$

Since, this bound already satisfies the constraint $\eta \leq 1$, the latter becomes inactive.

This gives us the $\eta_{t}(=\max \eta)$ as

$$
\eta_{t}=\frac{1}{\frac{N_{v}}{N_{t}-N_{v}} \frac{T_{s u c c}^{p}}{T_{\text {succ }}}+1}
$$

In summary, the value of $\eta_{t}$ depends on the ratio of victim to non-victim users, $T_{\text {succ }}^{p}$ (i.e., the average time required to transmit a data burst successfully in the contention free period) and $T_{\text {succ }}$ (i.e., the average time required to transmit a data burst successfully during the LTE-U ON period). Since, $T_{\text {succ }}^{p}$ and $T_{\text {succ }}$ depend on the Modulation and Coding Scheme (MCS) adopted, which in turn depends on the Signal to Noise Ratio (SNR) of the user, which again depends on the user location; the value of $\eta_{t}$ would finally be dependent on the ratio of victim and non-victim users and the user placement in the network.

Please note the inherent assumption that there exists at least one victim and non-victim user $\left(N_{v}>0\right.$ and $N_{n v}>0$ ). If a scenario has any of them zero, then the scheme degenerates to a normal DCF for $N_{v}=0$ or a complete CFP mechanism of operation for $N_{n v}=0$.

\subsection{Optimum CFP duration}

$T_{c f p}$ is defined as the minimum CFP duration required to serve victim users in a way that the average throughput of victim and non-victim users is same. Our approach to realize the minimum CFP duration 
is by observing the average throughputs of victim and non-victim users in the previous duty cycle period. Hence, we defined the updated CFP duration $\left(T_{c f p}\right)$ in terms of previous CFP duration $T_{c f p}^{o l d}$ as

$$
T_{c f p}=\frac{\Gamma_{n v}^{n e w}}{\Gamma_{v}^{n e w}} \cdot T_{c f p}^{o l d}
$$

In addition, the maximum value of $T_{c f p}$ cannot exceed the LTE-U OFF period, therefore, the final expression for calculating $T_{c f p}$ is given as

$$
T_{c f p}=\min \left(\frac{\Gamma_{n v}^{n e w}}{\Gamma_{v}^{n e w}} \cdot T_{c f p}^{o l d}, \text { LTE-U_OFF_Duration }\right)
$$

To quantify the fairness achieved by the above approach in calculating the CFP duration, we used the Jain's fairness index as follows.

$$
\text { fairness }=\frac{\left(\Gamma_{v}+\Gamma_{n v}\right)^{2}}{2 \times\left(\left(\Gamma_{v}\right)^{2}+\left(\Gamma_{n v}\right)^{2}\right)}
$$

where, $\Gamma_{v}$ and $\Gamma_{n v}$ are average throughputs of victim and non-victim users, respectively. Now, to prove the optimality of proposed scheme, we define the objective function as

$$
\text { max(fairness) }
$$

constraint on the fact that CFP duration cannot be more than the LTE-U OFF period.

$$
x \leq 1-\eta
$$

where $x$ is the fraction of CFP duration in an LTE-U duty cycle period and $\eta$ is the LTE-U ON fraction. Therefore, the objective function becomes

$$
J=\max \left(\frac{\left(\Gamma_{v}+\Gamma_{n v}\right)^{2}}{2 \cdot\left(\left(\Gamma_{v}\right)^{2}+\left(\Gamma_{n v}\right)^{2}\right)}\right)+\lambda(x-(1-\eta))
$$

Using the expressions of $\Gamma_{v}$ and $\Gamma_{n v}$ given in Eqns.25and26 for the proposed scheme for a DL only scenario, the above equation becomes,

$$
J=\frac{\left.\left((1-\eta-x) k_{1}+x k_{2}\right)+\left(\eta k_{3}+(1-\eta-x) k_{1}\right)\right)^{2}}{\left.2 \cdot\left((1-\eta-x) k_{1}+x k_{2}\right)^{2}+\left(\eta k_{3}+(1-\eta-x) k_{1}\right)^{2}\right)}+\lambda(x-(1-\eta))
$$

where $k_{1}=\frac{E[P]}{N_{t} \times T_{\text {succ }}^{\prime}}, k_{2}=\frac{E[P]}{N_{v} \times T_{\text {succ }}^{p}}$ and $k_{3}=\frac{E[P]}{\left(N_{t}-N_{v}\right) \times T_{\text {succ }}}$.

(Refer Section 6 for the explanation of each variable) By imposing the constraint that the maximum value of $\eta$ is less than $\eta_{t}$, (explained in Section 6.4), we have the condition, $x \leq 1-\eta$, always satisfied. This implies that the constraint is in-active and thus $\lambda=0$. Hence, we have

$$
J=\frac{\left(\left((1-\eta-x) k_{1}+x k_{2}\right)+\left(\eta k_{3}+(1-\eta-x) k_{1}\right)\right)^{2}}{2 \cdot\left(\left((1-\eta-x) k_{1}+x k_{2}\right)^{2}+\left(\eta k_{3}+(1-\eta-x) k_{1}\right)^{2}\right)}
$$

By differentiating with respect to $x$ and equating to zero, we get an extremely simplified expression for the CFP duration.

$$
x=\frac{\left(2(1-\eta)\left(k_{1} k_{2}-2 k_{1}^{2}\right)-2 \eta k_{1} k_{3}\right)\left(2(1-\eta) k_{1}+\eta k_{3}\right)-2\left(2(1-\eta)^{2} k_{1}^{2}+2(1-\eta) \eta k 3\right)\left(k_{2}-2 k_{1}\right)}{\left(2(1-\eta)\left(k_{1} k_{2}-2 k_{1}^{2}\right)-2 \eta k_{1} k_{3}\right)\left(k_{2}-2 k_{1}\right)-2\left(k_{2}^{2}+2 k_{1}^{2}-2 k_{1} k_{2}\right)\left(2(1-\eta) k_{1}+\eta k_{3}\right)}
$$




$$
\begin{aligned}
& \Longrightarrow x=\frac{\eta k_{3}\left((1-\eta) k_{1} k_{2}+\eta k_{3}\left(k_{1}-k_{2}\right)\right)}{k_{2}\left((1-\eta) k_{1} k_{2}+\eta k_{3}\left(k_{1}-k_{2}\right)\right)}=\frac{\eta k_{3}}{k_{2}} \\
& \Longrightarrow x=\frac{\eta N_{v} T_{\text {succ }}^{p}}{\left(N_{t}-N_{v}\right) T_{\text {succ }}}
\end{aligned}
$$

Therefore, the duration of CFP which achieves the maximum fairness is given by $\frac{\eta N_{v} T_{s u c c}^{p}}{\left(N_{t}-N_{v}\right) T_{\text {succ }}}$. Consequently, the throughput obtained with the maximally fair CFP duration would be

$$
\begin{gathered}
\bar{\Gamma}_{v}^{C C F}=\left(1-\eta-\frac{\eta N_{v} T_{\text {succ }}^{p}}{\left(N_{t}-N_{v}\right) T_{\text {succ }}}\right) \frac{1}{N_{t}} \frac{E[P]}{T_{\text {succ }}^{\prime}}+\frac{\eta N_{v} T_{\text {succ }}^{p}}{\left(N_{t}-N_{v}\right) T_{\text {succ }}} \frac{1}{N_{v}} \frac{E[P]}{T_{\text {succ }}^{p}} \\
=\left(1-\eta-\frac{\eta N_{v} T_{\text {succ }}^{p}}{\left(N_{t}-N_{v}\right) T_{\text {succ }}}\right) \frac{1}{N_{t}} \frac{E[P]}{T_{\text {succ }}^{\prime}}+\frac{\eta}{\left(N_{t}-N_{v}\right)} \frac{E[P]}{T_{\text {succ }}} \\
=(1-\eta) \frac{1}{N_{t}} \frac{E[P]}{T_{\text {succ }}^{\prime}}-\frac{\eta N_{v}}{\left(N_{t}-N_{v}\right) N_{t}} \frac{T_{\text {succ }}^{p}}{T_{\text {succ }}} \frac{E[P]}{T_{\text {succ }}^{\prime}}+\frac{\eta}{\left(N_{t}-N_{v}\right)} \frac{E[P]}{T_{\text {succ }}} \\
\bar{\Gamma}_{n v}^{C C F}=(\eta) \frac{1}{N_{t}-N_{v}} \frac{E[P]}{T_{\text {succ }}}+\left(1-\eta-\frac{\eta N_{v} T_{\text {succ }}^{p}}{\left(N_{t}-N_{v}\right) T_{\text {succ }}}\right) \frac{1}{N_{t}} \frac{E[P]}{T_{\text {succ }}^{\prime}} \\
=(\eta) \frac{1}{N_{t}-N_{v}} \frac{E[P]}{T_{\text {succ }}}+(1-\eta) \frac{1}{N_{t}} \frac{E[P]}{T_{\text {succ }}^{\prime}}-(\eta) \frac{N_{v}}{\left(N_{t}-N_{v}\right) N_{t}} \frac{T_{\text {succ }}^{p}}{T_{\text {succ }}} \frac{E[P]}{T_{\text {succ }}^{\prime}} \\
\bar{\Gamma}^{C C F}=\left(N_{v}\right) \bar{\Gamma}_{v}^{C C F}+\left(N_{t}-N_{v}\right) \bar{\Gamma}_{n v}^{C C F} \\
=(\eta) \frac{E[P]}{T_{\text {succ }}}+(1-\eta) \frac{E[P]}{T_{\text {succ }}^{\prime}}-(\eta) \frac{N_{v}}{\left(N_{t}-N_{v}\right) N_{t}} \frac{T_{\text {succ }}^{p}}{T_{\text {succ }}} \frac{E[P]}{T_{\text {succ }}^{\prime}}+(\eta) \frac{N_{v}}{\left(N_{t}-N_{v}\right)} \frac{E[P]}{T_{\text {succ }}}
\end{gathered}
$$

where $\bar{\Gamma}_{v}^{C C F}$ and $\bar{\Gamma}_{n v}^{C C F}$ are the optimal throughputs of the victim user and non-victim user, respectively and $\bar{\Gamma}^{C C F}$ is the total optimal throughput of the network for the proposed scheme.

\section{Performance Evaluation}

The system model described in Section 3 is simulated using MATLAB 35 with parameters given in Table 1. For simulation, we considered the same scenario as shown in Fig. 1. Results with DCF mechanism are compared with the CCF mechanism by simulating for 10 seconds and averaging over 10 different seed values. As the focus is to improve performance of victim users and Wi-Fi network, irrespective of duty cycle, the results are shown for the Wi-Fi network alone.

\subsection{DL only scenario}

Figs. 12 and 13 show the throughputs of victim user and non-victim users with the DCF and CCF mechanisms in DL only scenario, respectively. The figures show the simulation and analytical results with varying LTE-U ON Fraction $(\eta)$. The throughputs of victim user and non-victim user are not just higher (compared to DCF mechanism) but also are similar, thereby achieving fairness among Wi-Fi users, all the way from $\eta=0$ (LTE-U completely OFF) to $\eta=\eta_{t}$ (with $\eta_{t}=0.6$ ). We define $\eta_{t}$ as the maximum value of $\eta$ 
Table 1: Simulation Parameters

\begin{tabular}{|c|c|}
\hline Parameter & Value \\
\hline$C W_{\min }$ & 16 \\
\hline$C W_{\max }$ & 1024 \\
\hline PHY Header Size & 128 bits \\
\hline MAC Header Size & 272 bits \\
\hline ACK Size & 240 bits \\
\hline RTS Size & 288 bits \\
\hline Payload Size & 8148 bits \\
\hline $\begin{array}{l}\text { MAC Protocol Data Units } \\
\text { (MPDUs) }\end{array}$ & 4 \\
\hline Slottime & $9 \mu \mathrm{sec}$ \\
\hline CTStimeout & $50 \mu$ sec \\
\hline DIFS & $34 \mu \mathrm{sec}$ \\
\hline SIFS & $16 \mu$ sec \\
\hline Beacon Interval & $100 \mathrm{msec}$ \\
\hline$\alpha$ (Smoothing parameter) & 0.5 \\
\hline $\begin{array}{l}\text { Tx Power of AP, STAs } \\
\text { and eNB }\end{array}$ & $20 \mathrm{dBm}$ \\
\hline Operating Freq. & $5.3 \mathrm{GHz}$ \\
\hline System Noise & $-101 \mathrm{dBm}$ \\
\hline Channel Bandwidth & $20 \mathrm{MHz}$ \\
\hline eNB and AP Antenna Ht. & 10 meter \\
\hline User Antenna Ht. & 1 meter \\
\hline LTE-U ON Fraction $(\eta)$ & varied from 0.05 to 1 with step size of 0.05 \\
\hline Simulation time & $10 \mathrm{sec}$ \\
\hline Wi-Fi PHY Rates (Mbps) & $13,26,39,52,78,104,117,130$ \\
\hline Required SNR (in dB) & $5,7,9,13,17,20,22,23$ \\
\hline Traffic & Full buffer via saturated UDP flows \\
\hline Channel & No shadow/Rayleigh fading \\
\hline Path Loss Model & $36.7 \log 10(\mathrm{~d}[\mathrm{~m}])+22.7+26 \log 10($ freq[GHz] $) 9$ \\
\hline
\end{tabular}

for which throughput fairness is achievable. For $\eta>\eta_{t}$, the LTE-U OFF duration (i.e., $1-\eta$ ) is insufficient to provide the victim user with same throughput as that of the non-victim user, in spite of configuring the complete LTE-U OFF period as CFP.

Improvement in victim users throughput can be attributed to the use of CFP by the Wi-Fi AP to serve them, and improvement in non-victim users throughput can be imputed to the fact that Wi-Fi AP does not waste the channel in transmitting to the victim users during the LTE-ON duration and serves only the non-victim users. This further helps the Wi-Fi network to maintain throughput, especially in scenarios where $\eta>\eta_{t}$. From both simulation and analytical results, it is clearly observed that our CCF mechanism performs better in achieving fairness for $\eta \leq \eta_{t}$, and improving channel utilization as compared to the DCF mechanism, irrespective of $\eta$.

\subsection{Optimality of $T_{c f p}$ update}

To validate the optimality of the $T_{c f p}$ update procedure, we demonstrate the variation of CFP fraction (i.e., CFP fraction is the fraction of time AP is in CFP duration over the LTE-U duty cycle period) with time and compare its limiting value with the optimal CFP duration obtained. Fig. 14 shows the update in the CFP fraction for various LTE-U ON Fractions. Similarly, Fig. 15 compares the limiting behavior of 


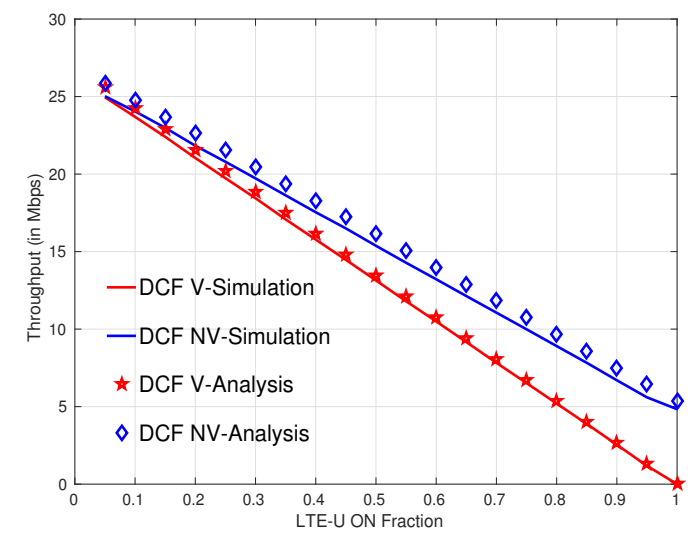

Figure 12: Throughputs of victim user and non-victim users for DCF mechanism with both simulation and analytical plots in DL only scenario for different LTE-U ON Fraction.

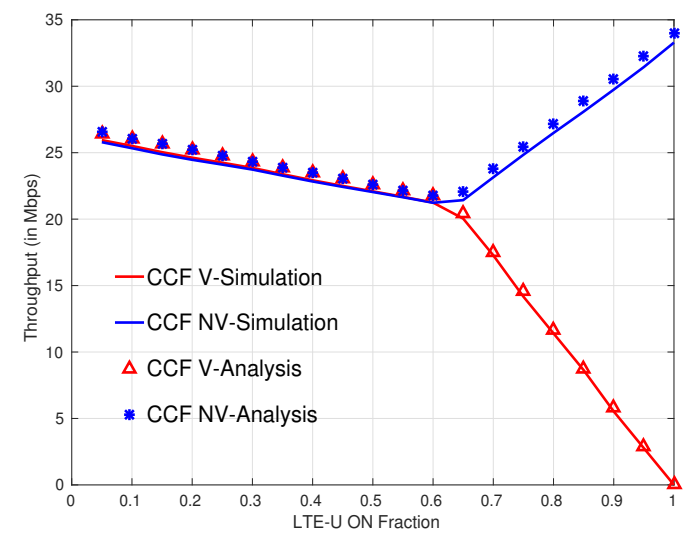

Figure 13: Throughputs of victim user and non-victim users for CCF mechanism with both simulation and analytical plots in DL only scenario for different LTE-U ON Fraction.

the CFP duration at $1^{\text {st }}$ second, $5^{\text {th }}$ second and the $10^{\text {th }}$ second, with the optimal value. The very small variation in CFP duration from $5^{t h}$ to $10^{t h}$ second proves that the update procedure is very effective in achieving the optimal value.

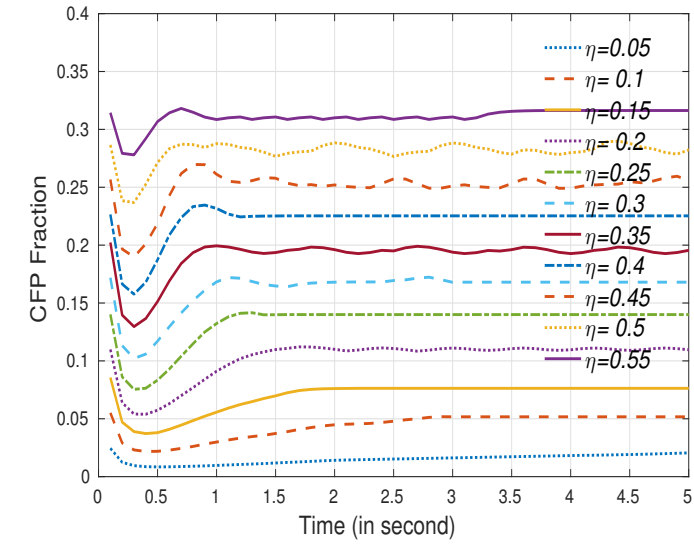

Figure 14: Variation of $T_{c f p}$ with time for various values of LTE-U ON Fraction. The update in the $T_{c f p}$ value is performed using the Eqn. 37.

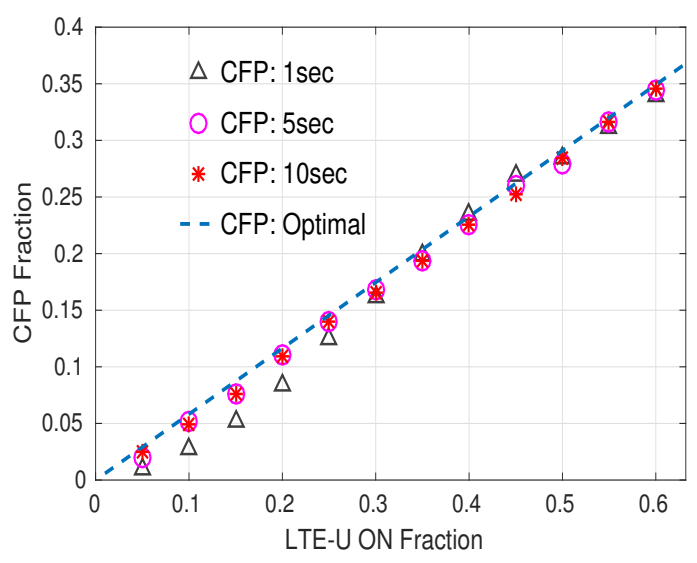

Figure 15: Variation of the limiting behavior of the CFP fraction at $1^{\text {st }}, 5^{\text {th }}$ and the $10^{t h}$ second, and the optimal value calculated using the optimization approach.

To further validate the proposed $T_{c f p}$ update procedure, we compare the throughput of the proposed scheme, obtained by using Eqn. (45) with the one obtained through simulations. Eqn. (45) is derived in Section 6.5 which essentially gives an expression for the optimum CFP duration, while the simulations use Eqn. (37), to update $T_{c f p}$

Since all the parameters that govern the throughput are fixed by external factors, except for the CFP duration (i.e., $T_{c f p}$ ), the throughput is a direct function of the CFP duration. The close match of the 
throughputs measured from simulation experiments with that obtained analytically (refer Fig. 16) confirms that the proposed scheme achieves optimality with regards to fairness among the victim and non-victim users, with Jain's fairness index being the fairness measure.

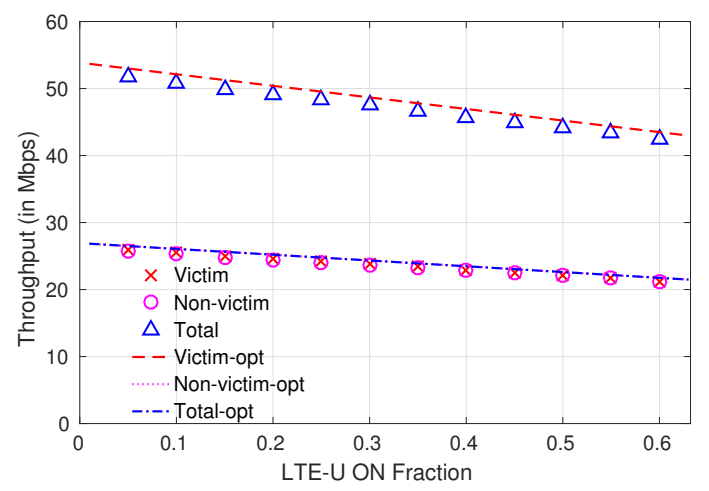

Figure 16: Variation in throughputs of victim users, non-victim users and total Wi-Fi network throughput for the CCF scheme with LTE-U ON Fraction. The CFP duration is optimal in the sense that is maximizes the fairness among the victim and non-victim users, with regards to the Jain's fairness index.

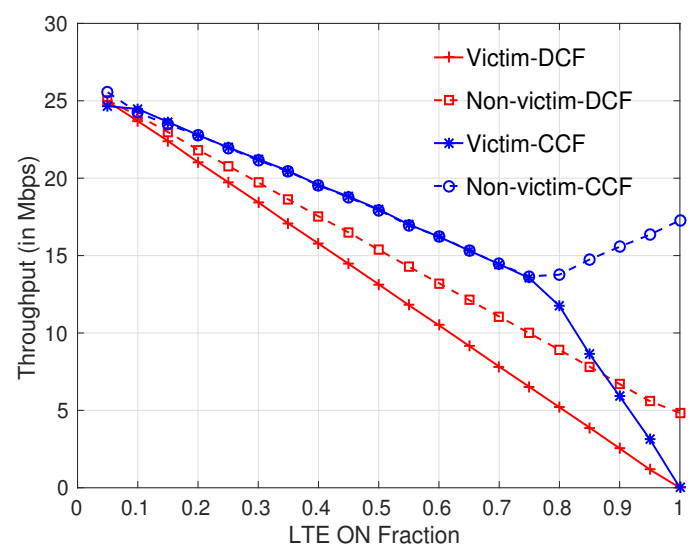

Figure 17: Throughputs of victim and non-victim users with varying LTE-U ON Fraction $(\eta)$ for CCF and DCF mechanisms in UL+DL scenario.

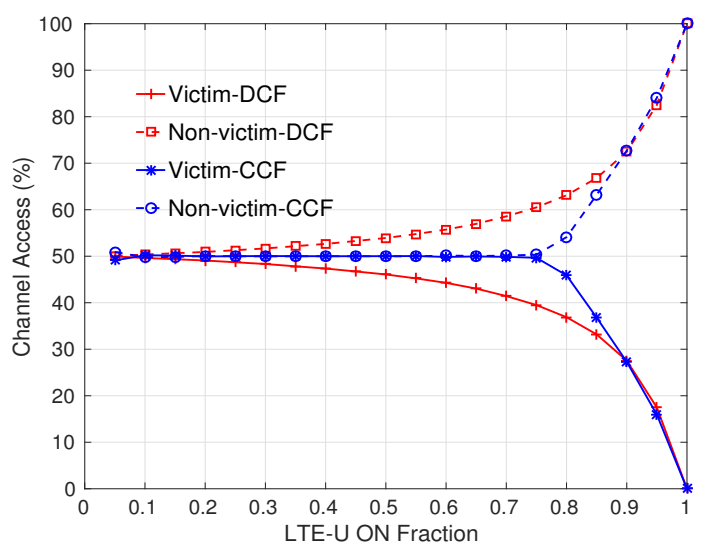

Figure 18: Successful channel access percentages of victim and non-victim users with varying LTE-U ON Fraction $(\eta)$ for $\mathrm{CCF}$ and DCF mechanisms in UL+DL scenario.

Figs. 17 and 18 show the performance of victim user and non-victim user - in terms of their throughputs and successful channel access percentages with varying $\eta$, respectively - for DCF and CCF mechanisms in UL+DL scenario. A similar trend in throughput, as with DL only traffic, can be observed ensuring fairness among the victim user and non-victim user for $\eta \leq \eta_{t}$, (with $\eta_{t}=0.75$ ). For $\eta>\eta_{t}$, the above conclusion, of LTE-U OFF period being insufficient for victim user still holds true. Successful channel access also being equal confirms that the throughput fairness was a result of equal access. Apart from fairness, for all $\eta<1$, a noticeable improvement in the throughput of victim user can be observed. 
Fig. 19 shows the performance of Wi-Fi AP in terms of DL throughput, DL opportunity, and successful DL transmission by varying LTE-U ON fraction. DL opportunity in case of DCF mechanism decreases as $\eta$ increases, since multiple retransmissions to victim user increases the average $\mathrm{BO}$ value. In the $\mathrm{CCF}$ mechanism, the Wi-Fi AP cleverly defers from transmitting to the victim user, during LTE-U ON period and avoids unnecessary increase in its average BO value, thereby preventing the decrease in DL opportunity of Wi-Fi AP. DL opportunity is now expected to be at $33 \%$ when $\eta$ is 0 (since all the three nodes get equal chance to access the channel, when LTE-U is OFF), and would increase to $50 \%$ when $\eta$ is 1 . A DL opportunity of $50 \%$ (at $\eta=1$ ) signifies that the non-victim user does not dominate the network and Wi-Fi AP still obtain equal chance to transmit as compared to the non-victim user.

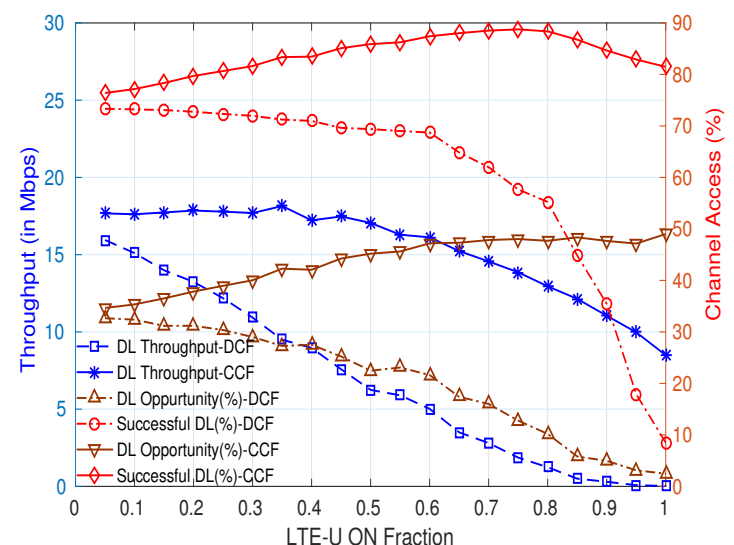

Figure 19: Performance of Wi-Fi AP in terms of DL throughput, DL opportunity, and successful DL percentage with varying $\eta$ for $\mathrm{CCF}$ and $\mathrm{DCF}$ in $\mathrm{UL}+\mathrm{DL}$ scenario.

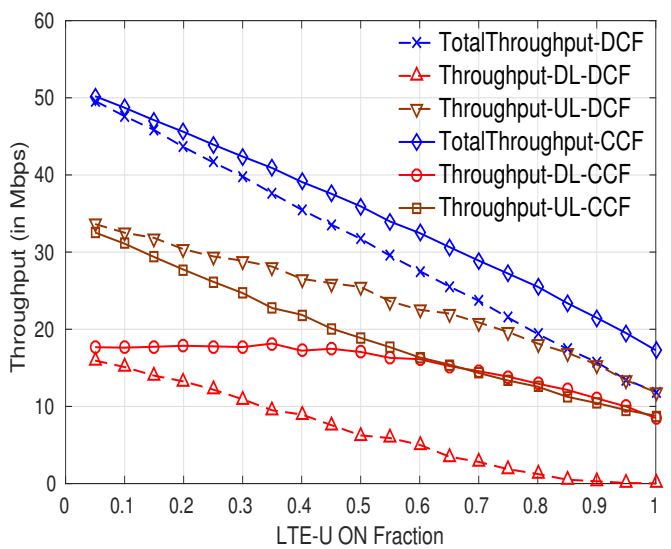

Figure 20: Performance of Wi-Fi AP in terms of UL throughput, DL throughput, and total throughput by varying $\eta$ for $\mathrm{CCF}$ and DCF mechanisms in UL+DL scenario.

Successful DL percentage in Fig. 19, which in the DCF mechanism was highly impacted by the retransmissions to the victim user, is now - with the CCF mechanism - just influenced by the collisions in the network, and hence is very high. Successful DL percentage increases with $\eta$ until $\eta_{t}$ (with $\eta_{t}=0.75$ ). An increase in LTE-U ON period demands for more CFP (to achieve fairness), and higher the CFP duration in a duty cycle, lower the collisions and hence successful DL percentage increases with $\eta$. For $\eta>\eta_{t}$, CFP duration is limited by LTE-U OFF period. Increasing $\eta$ more than $\eta_{t}$, will decrease the CFP duration due to a decrease in LTE-U OFF period. This constrained decrease in CFP duration results in a slight decrease in successful DL percentage (for $\eta>\eta_{t}$ ). Further, the DL throughput of the Wi-Fi AP has increased in comparison to the DCF mechanism, very distinguishably at higher $\eta$, which is clearly a result of increase in the DL opportunity and successful DL opportunity. The decrease in throughput with $\eta$ is unambiguous - due to the increased duration of LTE-U interference.

Fig. 20 shows how the UL throughput varies with the CCF mechanism and DCF mechanism. Clearly, the very high UL throughput - in DCF mechanism - is because the only user not affected by LTE-U is the non-victim user, and that user is able to send its data too frequently. Now, with the CCF mechanism, this perquisite enjoyed by the non-victim user was moderated. An expectation regarding DL throughput would be that it should be less than the UL (half in the above scenario), which is the case at very low duty cycle, but, as the duty cycle increases, this difference decreases. The decrease, in the considered scenario, can be attributed to the fact that CFP allows equal UL and DL opportunities, during the LTE-U OFF period and during the LTE-U ON period, the CCF mechanism prevents Wi-Fi AP from degrading its performance and hence allows it to equally compete with the non-victim user for the channel. Increase in $\eta$, further advocates both the above causes. 
Table 2: Wi-Fi Throughput Comparison using DCF and CCF mechanisms in UL+DL Scenario.

\begin{tabular}{|c|c|c|c|c|c|}
\hline LTE-U On Fraction $(\eta)$ & $\mathbf{0 . 2}$ & $\mathbf{0 . 4}$ & $\mathbf{0 . 6}$ & $\mathbf{0 . 8}$ & $\mathbf{1}$ \\
\hline DCF mechanism (in Mbps) & 41.62 & 33.57 & 25.54 & 17.46 & 11.88 \\
\hline CCF mechanism (in Mbps) & 45.58 & 39.09 & 32.46 & 25.50 & 17.27 \\
\hline
\end{tabular}

The total throughput of Wi-Fi network with the CCF mechanism has increased by $22.9 \%$, when averaged over $\eta$ compared to DCF mechanism, for the considered scenario as shown in Table 2 with minimum value of $9.51 \%$ at $\eta=0.2$ and maximum value of $45.37 \%$ at $\eta=1$.

\subsection{Varying percentage of victim users in the Wi-Fi network}

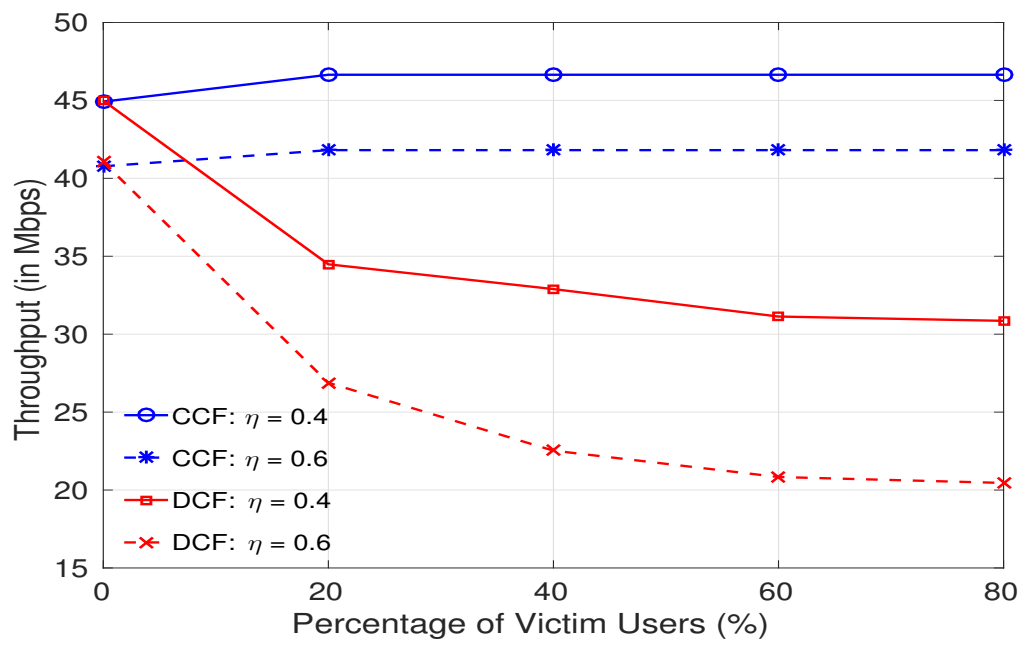

Figure 21: Throughput of the CCF and DCF mechanisms in DL only scenario for $\eta=0.4$ and $\eta=0.6$. Percentage of victim users is varied from 0 to $80 \%$ by keeping the total number of Wi-Fi users in the network as 10 .

To study the effect of percentage of victim users on the performance of Wi-Fi network in the DL only scenario, we varied the percentage of victim users in the network for both DCF and CCF mechanisms. As shown in Fig. 21, the performance of DCF mechanism degrades as the percentage of victim users increases in the network. This degradation is intelligently handled in the CCF mechanism by serving the victim and non-victim users based on ON and OFF periods of LTE-U. Hence, even if the percentage of victim users in the Wi-Fi network increases, the performance of CCF mechanism remains constant and always outperforms the DCF mechanism. In a trivial scenario, when the percentage of victim users in the network is zero (i.e., none of the users being victim), the performance of DCF mechanism is same as that of the CCF mechanism; as the CFP value is set to zero due to the absence of victim users in the network. When we increase the percentage of victim users in the network to $20 \%$, due to multiple unsuccessful retransmissions to victim users in the DCF mechanism, the average BO of Wi-Fi AP increases and hence Wi-Fi network's throughput reduces. Whereas the CCF mechanism avoids serving victim users in ON period and intelligently serves them in OFF period using CFP, thus outperforms the DCF mechanism. CCF mechanism performs slightly better in the presence of victim users than when no victim users because the presence of victim users enables PCF/HCF mode with CCF mechanism, where it polls and serves one of its users (namely, the victim users), as opposed to pure contention. This results in a slight increase in throughput of Wi-Fi network. Increasing percentage of victim users further decreases throughput of DCF mechanism, but the performance of CCF mechanism remains constant and it is on-par with that of the DCF mechanism. This shows the robustness of the CCF mechanism with varying percentage of victim users in the network. 


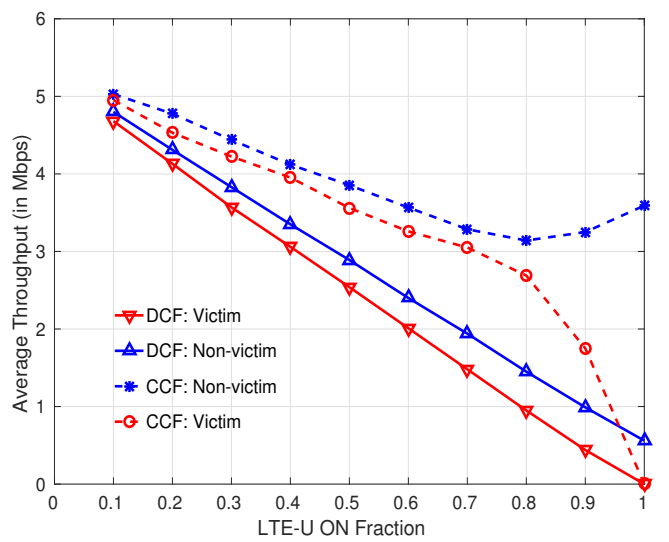

Figure 22: Average throughput of victim and non-victim users over 100 seeds for CCF and DCF mechanisms in DL with varying LTE-U ON Fraction $(\eta)$.

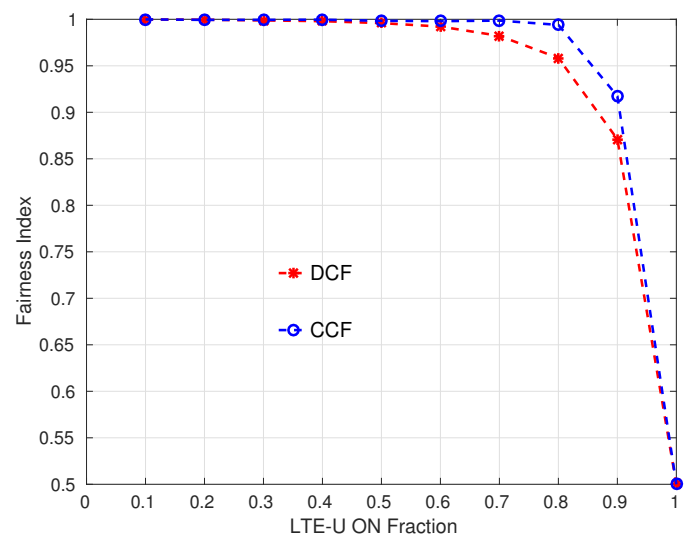

Figure 23: Throughput fairness of victim and non-victim users for DCF and CCF mechanisms in DL with varying LTE-U ON Fraction $(\eta)$.

In this experiments, we measure the average behavior of a Wi-Fi network in the DL only scenario for DCF and CCF mechanisms by varying positions of users. A total of $10 \mathrm{Wi}$-Fi users are placed at uniformly random within $20 \mathrm{~m}$ radius from the AP. This uniform random placement determined a configuration and thus all results are presented by averaging over 100 such configurations. Such placement varied the number of victim users in each run, with the average number of victim users being $33 \%$ in the experiment. The CCF mechanism serves these victim users in a round robin fashion in the CFP while for the DCF mechanism no changes are made in its functionality. Fig. 22 shows the average throughput of victim and non-victim users over 100 seeds for different $\eta$ values. From Fig. 22, it becomes clear that for all the values of $\eta$, the throughputs of both victim and non-victim users are considerably better in the CCF mechanism compared to the DCF mechanism.

In addition, the CCF mechanism also ensured a fair distribution of throughput among the victim and non-victim users. However this fairness is possible to achieve only until a threshold $\eta$, after which the CFP duration, which is indeed restricted by LTE-U OFF period, becomes insufficient to provide an identical throughput to victim users as that of non-victim users. Hence, after $\eta=0.7$ the throughput of victim users starts to decrease and the throughput of non-victim users starts to increase as ON period increases.

To study the throughput fairness between victim and non-victim users, we use the Jain's fairness index [34. The Jain's fairness index is a well-known indicator used to measure throughput fairness among users. The value of the Jain's fairness index ranges from 0 to 1 , where a value of 1 indicates maximum fairness and a 0 indicates minimum fairness. Fig. 23 shows the Jain's fairness index between the average throughput of victim and non-victim users for both CCF and DCF mechanisms. It can be observed from the figure that the fairness of CCF and DCF mechanisms are almost same. Hence, using CCF mechanism we are able to improve the performance of victim and non-victim users without making any compromise on fairness between victim and non-victim users.

Fig. 24 shows the throughput CDFs of DCF and CCF mechanisms for different $\eta$. A trivial observation is that the throughput of Wi-Fi at lower $\eta$ is higher than that with higher $\eta$ for both CCF and DCF mechanisms. This is because the increase in ON period of LTE-U decreases the Wi-Fi's opportunity to access the medium and serve its users. Hence, increase in $\eta$ decreases the throughput for both the mechanisms. However, for all $\eta$ values, the CCF mechanism outperforms DCF mechanism. The improvement is a combined result of 


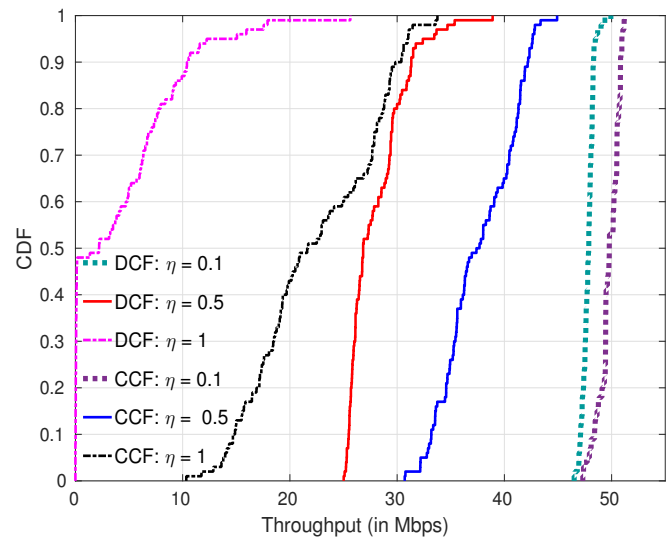

Figure 24: Throughput CDF of Wi-Fi network in the CCF and DCF mechanisms in DL only scenario for different LTE-U ON Fraction $(\eta)$. ' 10 ' users are placed uniformly random, with each placement determining a configuration and throughputs are collected for 100 such configurations.

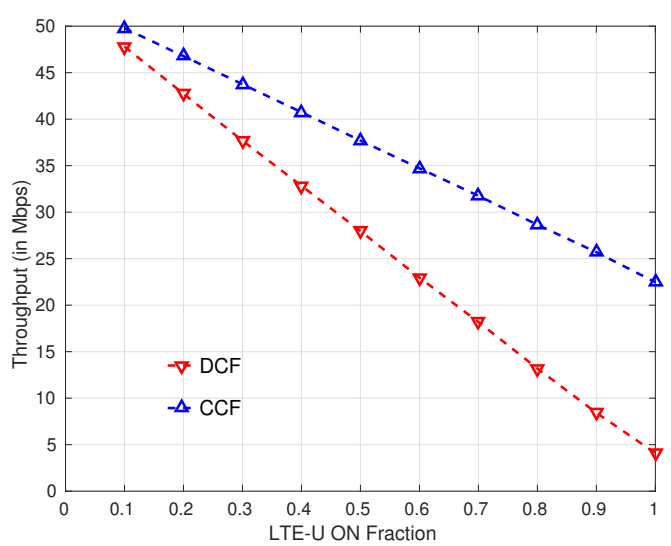

Figure 25: Average throughput of Wi-Fi network throughput over 100 seeds for CCF and DCF mechanisms in DL scenario with varying LTE-U ON Fraction $(\eta)$.

intelligently serving Wi-Fi users and efficiently utilizing the unlicensed spectrum by avoiding unnecessary multiple retransmissions in the LTE-U ON period.

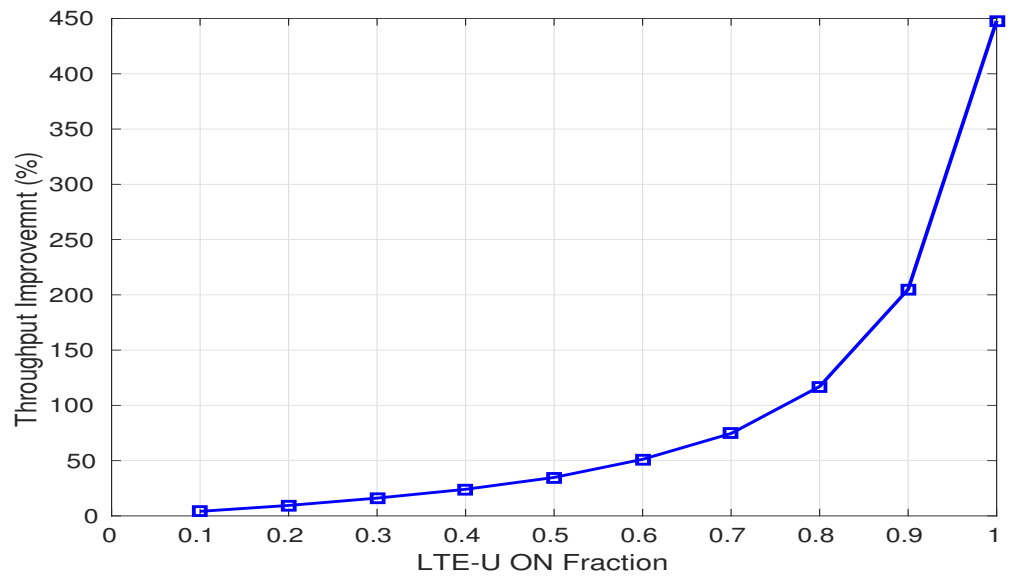

Figure 26: Wi-Fi network throughput improvement (\%) in the CCF mechanism over DCF mechanism in DL only scenario with varying LTE-U ON Fraction $(\eta)$.

Fig. 25 shows the average throughput of Wi-Fi network with 10 users for 100 different configurations (i.e., for 100 different random placement of users). Results are collected for both the mechanisms for different $\eta$ values. From figure it is clear that CCF, apart from providing a boost in victim user performance, outperforms DCF mechanism in terms of network throughput as well, for all values of $\eta$. The simultaneous improvement in the victim users performance as the well the network performance makes it clear that the improvement provided be CCF was not simply achieved by compromising throughput of the non-victim users. Instead it was a proficient effort towards intelligently serving the disadvantaged users. Fig. 26 shows the throughput improvement percentage (\%) of CCF mechanism over DCF mechanism. Although 
the improvement is only $4 \%$ for $\eta=0.1$, but this is just the minimum improvement. As the $\eta$ increases the improvement of CCF over the DCF mechanism escalates significantly. In the case, where LTE-U and Wi-Fi share the channel equally (i.e., at $\eta=0.5$ ) the improvement of CCF over DCF is $35 \%$, which reaches an exorbitant value of $448 \%$ for $\eta=1$. Therefore, with increase in $\eta$, the performance of CCF mechanism increases compared to DCF mechanism. Packet losses and multiple retransmissions in DCF mechanism are the major factors for its performance degradation, which gets worsened with increase in $\eta$. Whereas CCF mechanism intelligently uses CFP and CP of IEEE 802.11 standard and thereby avoids these losses and shows better improvement.

\subsection{Performance evaluation in mobile scenario}

To understand the behavior of the proposed scheme in a scenario involving mobile users, we attached 10 Wi-Fi users to the Wi-Fi AP. These users were placed uniformly in a circle of radius $10 \mathrm{~m}$ with AP being at its center. To make users mobile, we imposed the random mobility model, which allows users to assume a random velocity and move in a randomly selected direction for a specified interval of time. To make the simulations experiments more realistic, we restricted the velocity to a maximum value of $5 \mathrm{~km} / \mathrm{h}$. Further, the LTE-U eNB is following ON-OFF cycles with LTE-U ON Fraction of 0.5.

As earlier, the LTE-U eNB is placed at a distance $20 \mathrm{~m}$ from the Wi-Fi AP, and consequently we have a few of the Wi-Fi users as victims. However, now because of mobility of these users, the number of victim users in the network keeps varying. Fig. 27 captures one such instance where the number of victim users varies from a single user to 4 users over 40 sec interval in the Wi-Fi network.

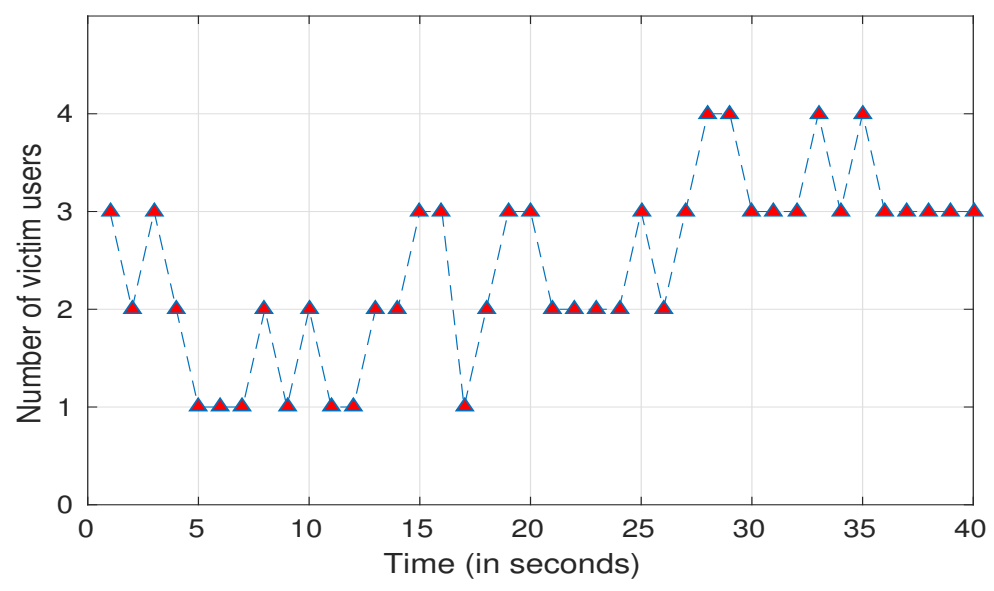

Figure 27: Variation in number of victim users over time due to user mobility.

To prove the robustness of the proposed scheme, we perform a throughput comparison for the above 'mobility based' scenario, between the CCF and the DCF schemes. Fig. 28 shows the variation in the aggregated throughputs of victim and non-victim users for the two schemes. Clearly, the total throughput of the victim as well the non-victim employing the CCF approach is significantly better than the ones using the DCF mechanism. This proves that the proposed scheme can easily accommodate any changes in the number of victim users, resulting from the movement of users in the network.

In fact, since the performance of the scheme is predominantly dependent on the number of victim users, other factors including but not restricted to fading, abrupt environmental changes and movement of users that results in a change in number of victim users would give the same behavior as reported above and 


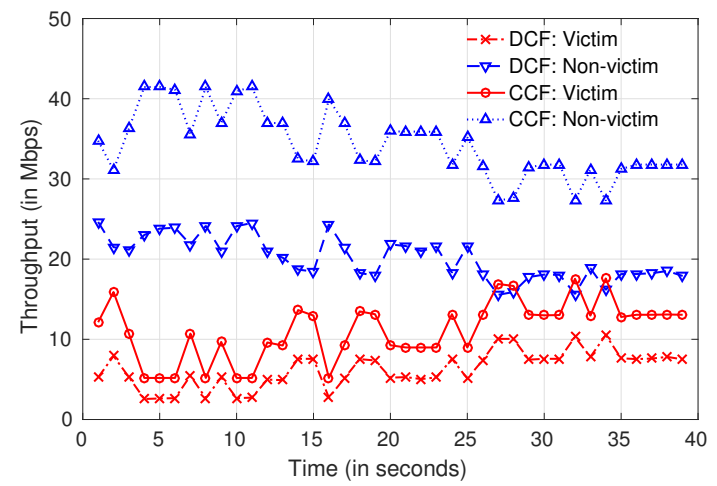

Figure 28: Variation in throughputs of victim and non-victim users in mobile scenario.

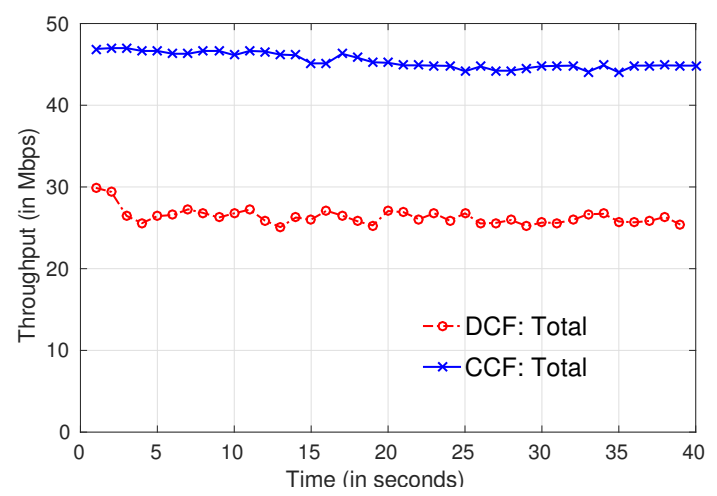

Figure 29: Total network throughput comparison between $\mathrm{CCF}$ and DCF mechanisms.

can easily accommodated by the proposed scheme. This advantage can be attributed to the update based approach of the proposed scheme for determining the optimum CFP duration.

Furthermore, Fig. 29 demonstrates the improvement achieved in terms of the total Wi-Fi network throughput. This substantial gain clearly proves the upper hand which the proposed has over the DCF based approach in terms of efficient spectrum utilization as well.

\section{Conclusions}

In this paper, we focused on improving the performance of Wi-Fi network in the presence of LTE-U network. We have observed that the performance of Wi-Fi, depending on the interference from LTE-U eNB, can degrade to a large extent. We found that, in cases when LTE-U transmissions partially affect Wi-Fi network, not only the fairness among Wi-Fi users is compromised, but also the Wi-Fi AP's throughput is affected. To address this issue, we proposed a scheme based on PCF/HCF namely CCF, which can be easily adopted by $\mathrm{Wi}-\mathrm{Fi}$, to ensure fairness among its users and to improve the performance of Wi-Fi network. In the considered scenario with uniform random placement for 100 seeds, the minimum improvement observed for the CCF mechanism over DCF mechanism is $4 \%$ for $\eta=0.1$ and when LTE-U and Wi-Fi share the channel equally (i.e., at $\eta=0.5$ ) the improvement is $35 \%$. The maximum improvement observed is $448 \%$ at $\eta=1$. The efficacy of the scheme is shown by sufficient analysis and simulation experiments.

\section{ACKNOWLEDGMENT}

This work was supported by the project "Converged Cloud Communication Technologies", Deity, Govt. of India.

\section{REFERENCES}

[1] Cisco Visual Networking Index: global mobile data traffic forecast update, 2013-2018, Cisco White Paper (Feb 2014).

[2] 3GPP, TSGRAN; Study on Licensed-Assisted Access to Unlicensed Spectrum, Tech. Rep. TR 36.889 V13.0.0 (June 2015).

[3] ETSI, Broadband Radio Access Networks (BRAN); 5 GHz high performance RLAN; Harmonized EN covering the essential requirements of article 3.2 of the R\&TTE Directive, in: ETSI EN 301893 V1.8.1, 2015.

[4] LTE in Unlicensed Spectrum: Harmonious Coexistence with Wi-Fi, Qualcomm White Paper (June 2014). 
[5] C. Cano, D. J. Leith, Unlicensed LTE/WiFi Coexistence: Is LBT Inherently Fairer Than CSAT?, in: IEEE International Conference on Communications (ICC), July 2016.

[6] LTE-U SDL Coexistence Specifications, LTE-U Forum [Online]. Available: http://www.Iteuforum.org/documents.html (2015).

[7] A. K. Sadek, Carrier sense adaptive transmission (CSAT) in unlicensed spectrum, US Patent (Nov. 8 2016).

[8] M. R. Khawer, J. Tang, F. Han, usICIC: A Proactive Small Cell Interference Mitigation Strategy for Improving Spectral Efficiency of LTE Networks in the Unlicensed Spectrum, IEEE Transactions on Wireless Communications 15 (3) (2016) $2303-2311$.

[9] 3GPP, 3GPP-TSG-RAN-WG1; Evolved Universal Terrestrial Radio Access (E-UTRA), Tech. Rep. TR 36.814 V9.0.0 (March 2010).

[10] R. Zhang, M. Wang, L. X. Cai, Z. Zheng, X. Shen, L.-L. Xie, LTE-unlicensed: the future of spectrum aggregation for cellular networks, IEEE Wireless Communications 22 (3) (2015) 150-159.

[11] Nokia LTE for unlicensed spectrum, White Paper (June 2014).

[12] C. Cano, D. Lopez-Perez, H. Claussen, D. J. Leith, Using LTE in Unlicensed Bands: Potential Benefits and Coexistence Issues, IEEE Communications Magazine 54 (12) (2016) 116-123.

[13] LTE and Wi-Fi in Unlicensed Spectrum: A Coexistence Study, Nihar Jindal and Donald Breslin (2016).

[14] F. M. Abinader, E. P. Almeida, F. S. Chaves, A. M. Cavalcante, R. D. Vieira, R. C. Paiva, A. M. Sobrinho, S. Choudhury, E. Tuomaala, K. Doppler, et al., Enabling the coexistence of LTE and Wi-Fi in unlicensed bands, IEEE Communications Magazine 52 (11) (2014) 54-61.

[15] A. Babaei, J. Andreoli-Fang, Y. Pang, B. Hamzeh, On the impact of LTE-U on Wi-Fi performance, International Journal of Wireless Information Networks 22 (4) (2015) 336-344.

[16] A. M. Cavalcante, E. Almeida, et al., Performance evaluation of LTE and Wi-Fi coexistence in unlicensed bands, in: IEEE 77th Vehicular Technology Conference (VTC Spring), 2013.

[17] T. Nihtilä, V. Tykhomyrov, O. Alanen, et al., System performance of LTE and IEEE 802.11 coexisting on a shared frequency band, in: IEEE Wireless Communications and Networking Conference (WCNC), 2013.

[18] E. Almeida, A. M. Cavalcante, et al., Enabling LTE/WiFi coexistence by LTE blank subframe allocation, in: IEEE International Conference on Communications (ICC), 2013.

[19] H. Zhang, X. Chu, W. Guo, S. Wang, Coexistence of Wi-Fi and heterogeneous small cell networks sharing unlicensed spectrum, IEEE Communications Magazine 53 (3) (2015) 158-164.

[20] LTE-U Forum , [Online]. Available: http://www.Iteuforum.org/ (2015).

[21] LTE-U Technical Report, LTE-U Forum [Online]. Available: http://www.lteuforum.org/documents.html (2015).

[22] UE Minimum Requirements for LTE-U SDL, LTE-U Forum [Online]. Available: http://www.1teuforum.org/documents. html (2015).

[23] eNB Minimum Requirements for LTE-U SDL, LTE-U Forum [Online]. Available: http://www . Iteuforum.org/documents . html (2015).

[24] Q. Chen, G. Yu, H. Shan, A. Maaref, G. Y. Li, A. Huang, Cellular meets WiFi: Traffic offloading or resource sharing?, IEEE Transactions on Wireless Communications 15 (5) (2016) 3354-3367.

[25] Q. Chen, G. Yu, A. Maaref, G. Li, A. Huang, Rethinking Mobile Data Offloading for LTE in Unlicensed Spectrum, IEEE Transactions on Wireless Communications 15 (7) (July 2016) 4987 - 5000.

[26] 802.11-2012-IEEE Standard for Information technology-Telecommunications and information exchange between systems Local and metropolitan area networks-Specific requirements Part 11: Wireless LAN Medium Access Control (MAC) and Physical Layer (PHY) Specifications, IEEE Standards Association and others, Available: http://standards.ieee.org/ about/get/802/802.11.html (2012).

[27] L. B. Jiang, S. C. Liew, Improving throughput and fairness by reducing exposed and hidden nodes in 802.11 networks, IEEE Transactions on Mobile Computing 7 (1) (2008) 34-49.

[28] K. Nishide, H. Kubo, R. Shinkuma, T. Takahashi, Detecting hidden and exposed terminal problems in densely deployed wireless networks, IEEE Transactions on Wireless Communications 11 (11) (2012) 3841-3849.

[29] C. Huang, C.-T. Lea, A. K.-S. Wong, A joint solution for the hidden and exposed terminal problems in csma/ca wireless networks, Computer networks 56 (14) (2012) 3261-3273.

[30] A. Jayasuriya, S. Perreau, A. Dadej, S. Gordon, Hidden vs exposed terminal problem in ad hoc networks, Ph.D. thesis, ATNAC 2004 (2004).

[31] M. Ismail, W. Zhuang, A distributed multi-service resource allocation algorithm in heterogeneous wireless access medium, IEEE Journal on Selected Areas in Communications 30 (2) (2012) 425-432.

[32] A. R. Elsherif, W.-P. Chen, A. Ito, Z. Ding, Resource allocation and inter-cell interference management for dual-access small cells, IEEE Journal on Selected Areas in Communications 33 (6) (2015) 1082-1096.

[33] B. P. Crow, I. Widjaja, L. Kim, P. T. Sakai, IEEE 802.11 wireless local area networks, IEEE Communications magazine 35 (9) (1997) 116-126.

[34] R. Jain, D.-M. Chiu, W. Hawe, A quantitative measure of fairness and discrimination for resource allocation in shared computer systems.

[35] MATLAB, version 9.0.0.341360 (R2016a), The MathWorks Inc., Natick, Massachusetts, 2016. 